\title{
Les tiques des animaux domestiques de l'Afrique Occidentale Française
}

\author{
par P. C. MOREL
}

SOMMAIRE

I. Introduction

153

II. Tiques en Afrique Occidentale

Française .................. 154

A propos de la présentation....... 154

Ixodidae ...................... 155

$1^{\circ}$ Amblyomma variegatum....... 155

$2^{\circ} \quad-\quad$ paulopunctatum .. 159

30 $\quad-\quad$ splendidum ...... 159

$4^{\circ} \quad-\quad$ nuttalli ........ 159

$5^{\circ}$ Boophilus decoloratus ....... 159

$6^{\circ}$ - annulatus (= congolensis)........... 163

$7^{\circ}$ Haemaphysalis aciculifer ..... 164

$8^{\circ} \quad-\quad h o o d i . . . \ldots . .164$

$90 \quad$ - $\quad$ leachi leachi ... 164

$10^{\circ} \quad-\quad$ parmata ...... 165

$11^{\circ}$ Hyalomma dromedarii ........ 165

$12^{\circ}-$ excavatum ....... 166

$13^{\circ}-$ impeltatum $=$ brumpli)......... 168

$14^{\circ} \quad-\quad$ impressum........ 170

\section{INTRODUCTION}

Les connaissances sur les Ixodidés d'A.O.F. ont été longtemps fragmentaires. Quelques références, en général peu précises, de Neumann constituent tout ce qu on en sait jusquen 1940-45. A cette époque, divers auteurs ont entrepris la révision systématique de certains genres importants en ce qui concerne les tiques du bétail : Zumpt pour le genre Rhipicephalus, $15^{\circ}$ Hyalomma rufipes .......... 170

$16^{\circ}-$ truncatum $(=$ transiens) ......... 171

$17^{\circ}$ Espèces du genre Ixodes ..... 173

$18^{\circ}$ Rhipicephalus capensis longus .. 173

$19^{\circ} \quad-\quad$ compositus..... 173

$20^{\circ}-\quad-\quad$ evertsi evertsi... 173

$21^{\circ} \quad-\quad$ (Pterygodes) fulvus......... 175

$22^{\circ} \quad-\quad$ pravus........ 175

$23^{\circ}-\quad-\quad$ sanguineus ...... 175

$24^{\circ}-$ simus........ 178

$25^{\circ} \quad-\quad$ tricuspis $(=l u-$ nulatus) $\ldots \ldots .180$

$26^{\circ}-\quad-\quad$ ziemanni ...... 180 Argasidae ................... 181

$27^{\circ}$ Argas persicus ............ 181

$28^{\circ}$ - reflexus (ou espèce voisine) ............ 182

$29^{\circ}$ Ornithodoros foleyi ........ 182

$30^{\circ}-$ savignyi....... 182

III. Répartition des tiques en A.O.F. en fonction des zones climatiques 183

IV. Bibliographie .............. 185

Delpy pour le genre Hyalomma (1). Les chercheurs eurent alors à leur disposition de sûrs instruments de travail. Il convient de remarguer que beaucoup d'études sur les Ixodidés ont été entreprises à ce moment en divers territoires

(1) Ces genres présentaient en effet des difficultés particulières du point de vue de la détermination des espèces. Sur d'autres genres, on possédait déjà les monographies de Nuttall et son école (Hacmuphysalis, Amblyarma). 
d'Afrique : Guinée Portuge ise, Nigeria, Carmeroun, Sudan, Afrique Orientale Anglaise, Mozambique, Angola. En Afrique du Sud les recherches sur les tiques et les maladies qu'elles transmettent, commencées par A. Theiler et Lounsbury au début de ce siècle, ont été poursuivies jusqu'à nos jours par Bedford et Miss $\mathrm{G}$. Theiler. Pour l'Afrique française, Rousselot (1948, 1951, 1953) a publié les premières données précises sur les tiques du bétail, malheureusement limitées à une seule région en ce qui concerne l'A.O.F. (Bamako-Ségou). Fiasson (1943) traitait des tiques d'A.E.F.

En raison de l"importance de ces arthropodes, soit par leur abondance même et leur action prédatrice directe, soit par les maladies transmises (protozoaires, rickettsies, bactéries, ultravirus) ou leurs toxines propres, il a semblé nécessaire de mener en A.O.F. une enquête sur la répartition géographique précise des diverses espèces, enquête absolument indispensable si on souhaite s'appliquer par la suite à l'étude des affections transmises ou si on veut mettre en cuvre, d'une manière raisonnée, un plan de lutte contre ces parasites. En entreprenant un tel travail, nous ne faisons d'ailleurs que nous conformer aux recommandations formulées à l'issue de la Réunion de juillet 1955 par le Bureau Interafricain des Maladies Epizootiques (I.B.E.D.).

La note présentée ici constitue le résultat des prospections effectuées pendant un séjour de deux ans. Une partie du matériel a été recueillie par nous-même au cours de tournées Le reste des données a été fourni par les collections envoyées au laboratoire. Nous avons essayé d'unifier par des notices préalables le mode de recherche, de récolte, etc... Malgré la diversité inhérente au nombre des collecteurs, nous pouvons commencer à coordonner les premiers résultats. Sans l'aide obligeante des Chefs de Service de l'Elevage dans la diffusion des demandes d'envoi, sans la compréhension et le concours actif de tous les membres du Service de l'Elevage, ce travail n'aurait pu être mené à bien. Qu'il nous soit permis de les remercier tous ici.

Nous avons également consulté les collections de l'Institut Français d'Afrique Noire, grâce à l'obligeance de MM. Villiers, Dekcyser et Condamin. M. Abonnenc nous a communiqué les tiques de la Faculté de Médecine de Dakar. MM. Hamon, Rickenbach et Ovazza, du Service Général d'Hygiène Mobile et de Prophylaxie de l'A.O.F. nous ont amicalement confié le matériel d'lxodidés qu ils récoltaient, ainsi que MM. Doucet et Adam, de l'Office de la Recherche Sciertifique et Technique d'Outre-Mer à Adiopodcumé (Abidjan).

Nous avons profité d'un congé pour examiner à Paris les collections de l'Institut Pasteur (Laboratoire de M. le Professeur Roubaud et du Dr. Colas-Belcour), du Muséum de Paris (Laboratoire du Dr. M. André), de la Faculté de Médecine (Collection Brumpt : Laboratoire de M. le Professeur Galliard et du Dr. Chabaud). Une mission à Toulouse nous a permis de consulter les collections de Neumann à l'Ecole Vétérinaire (Laboratoire de M. le Professeur Brizard). Nous avons pu également y prendre connaissence des nombreux renseignements que Neumann avait consignés dans ses dossiers.

Nous nc traiłons ici que des espèces parasites des animaux domestiques. Evidemment nous citons les trouvailles de ces espèces sur animaux sauvages, et réciproquement les références d'espèces parasites d'animaux sauvages occasionnellement trouvées sur animaux domestiques. Les tiques d'animaux sauvages feront l'cbjet d'une note séparée.

L'enquête en cours n'est pas terminée. Le lecteur s'apercevra que pour certaines régions il existe des lacunes importantes. Pour d'autres régions nous ne connaissons que quelques récoltes, faites à un seul moment de l'année. Tous les renseignements que nous fournissons aujourd'hui ont besoin d'être complétés. Il convient donc d'espérer que cette enquête pourra se poursuivre encore, avec le concours de tous ceux qui déjà nous ont aidé à réunir les données de ce premier rapport.

\section{TIQUES EN \\ AFRIQUE OCCIDENTALE FRANÇAISE}

\section{A propos de la présentation :}

L'étendue de ce rapport ne nous a pas permis d'indiquer à l'occasion de chaque récolte, la date du prélèvement, les stades présents, l'importance de cette récolte, les numéros de référence à la collection du laboratoire, ni les noms des collecteurs. Nous avons donc réduit les renseignements à l'essentiel, c'est-à-dire les localités et les hôtes.

Il est entendu que lorsque l'hôte n'est pas indiqué, il s'agit de bovin (bouf sans bosse ou zébu), sauf dans le cas de $H$. dromedarii, dont l'hôte implicite est le dromadaire. 
Seuls les stades larvaires et nymphaux sont signalés $(l, n)$; l'absence d'indication à ce sujet traduit la présence des formes adultes seulement (sauf en ce qui concerne la présence d'Amblyomma variegatum chez des bovins,. sur lesquels les immatures sont presque aussi fréquents que les adultes, surtout en saison sèche).

Dans le cas des animaux sauvages, nous ne donnons pas la désignation latine. Tous les renseignements à ce sujet paraîtront ultérieurement.

Voici la liste des abréviations utilisées, mentionnant les diverses collections consultées autres que celle du Laboratoire de l'Elevage de Dakar:

CBpt Collection Brumpt, Faculté de Médecine de Paris,

$\mathrm{CNm}$ Collection Neumann, à Toulouse,

IFAN Collection de l'Institut Français d'Afriquie Noire,

MHNP Muséum d'Histoire Naturelle de Paris, IPP Institut Pasteur de Paris (laboratoire d'Entomologie),

SCHMP Service Général d'Hygiène Mobile et de Prophylaxie à Bobo Dioulasso,

Neumann, in tab. (= in tabulis) : renseignements trouvés dans les fiches et dossiers de Neumann, à Toulouse.

\section{IXODIDAE}

\section{$1^{\circ}$ Amblyomma variegatum (Fabricius, 1794).}

Les espèces du genre Amblyomma sont le plus généralement liées aux animaux sauvages et cette spécificité est plus ou moins étroite. Cependant certaines espèces peuvert accidentellement parasiter les animaux domestiques du même groupe zoologique que les hôtes sauvages.

$A$. variegatum fait exception à cette règle en apparence : dans toutes les collections il est rapporté beaucoup plus abondamment sur animaux domestiques que sur animaux sauvages. En fait il doit s'agir d'une espèce qui s'est adaptée secondairement au bétail, et tellement bien que c'est la tique numériquement la plus importante, en ce qui concerne l'Afrique occidentale.

\section{BIOLOCIE}

C'est une tique à trois hôtes. Les immatures se gorgent surtout sur les animaux sauvages, oiseaux, reptiles, rongeurs, petits carnivores, mais aussi sur le chien, le chat, les petits et grands ruminants, le cheval, l'âne. L'homme est très fréquemment attaqué par les larves. Les stades immatures sont donc véritablement ubiquistes. Les adultes ont de leur côté une préférence marquée pour les grands mammifères. domestiques et sauvages (antilopes, phacochères, etc...) Il n'est pas rare d'en-rencontrer sur les carnivores.

Les adultes se fixent surtout, pour ce qui est des bovins, sur les régions inférieures : fanon, ars, scrotum, mamelles, périnée, paturons; ils enfoncent leur rostre très profondément (l'arrachage en est difficile) et provoquent souvent un abrès au point de fixation. Les endroits lésés présentent des cicatrices durables, dont la confluence peut faire penser à certaines dermites (à la streptothricose par exemple). Ces lésions peuvent être également le point de dépôt d'œufs de Diptères. A Djougou nous avons pu constater une myiase à Chrysomyia sp. sur la mamelle d'une vache de 2 ans, probablement secondaire à une plaie de fixation d'Amblyomma, nombreux en particulier sur cette vache. Chez les petits ruminants et les équidés, les localisations sont semblables.

Les nymphes n'ont pas de lieux de fixation préférentiels; on peut les trouver sur tout le corps, mais surtout sur l'encolure et les membres. Chez les petits ruminants on les rencontre surtout dans les oreilles, au périnée et entre les doigts. Les larves sont fixées sur tout le corps, souvent massivement dans l'oreille et sur la tête.

Le temps du repas de la femelle dure au minimum une semaine, le plus souvent jusqu'à deux semaines. Les mâlcs semblent pouvoir demeurer plus longtemps fixés. Les nymphes et les larves se gorgent en moyenne en une semaine. La femelle gorgée peut atteindre un volume considérable.

Fn région sahélienne et soudanienne il semble qu'il n'y ait qu'une génération par an : on assiste à une sorte d'explosion des adultes à la fin de la saison sèche et au début de l'hivernage. Le reste du temps on trouve surtout des immatures, et presque uniquement ces derniers en saison froide (janvier-février). En région guinéenne on observe des adultes toute l'année, en moindre importance numérique il est vrai durant la saison fraîche. Il est possible qu'en cette région il y ait plusieurs générations par an (deux sont probables) de mars à novembre, mais alors la 
sortie des adultes n'y a pas l'allure massive et brutale qu' elle a plus au nord.

\section{HYGROPHILIE}

Les exigences de l'espèce à ce point de vue semblent être peu strictes, car on la rencontre en $\Lambda . O . F$. à partir de l'isohyète de $500 \mathrm{~mm}$, jusqu'en forêt où elle est cependant un peu moins abondante qu'en savane (peut-être du fait des moindres rassemblements du bétail). En fait, sa plus grande fréquence se place entre les isohyètes de 500 et $1.500-2.000 \mathrm{~mm}$, où elle dépasse alors, du point de vue numérique, toutes les autres tiques. On en observe de très nombreux adultes en hivernage sur les herbes de savane (à 50-80 cm du sol) ou par terre. L'espèce ne semble pas subsister dans le nord de la région sahélienne (où règnent les Hyalomma).

\section{HOTES}

En premier licu les bovins, zébus, puis les petits ruminants, les équidés et porcins. Les carnivores domestiques sont surtout attaqués par les nymphes et larves.

En Afrique occidentale les hôtes sauvages pour les adultes sont les suivants : guib, bubale, hippotragues, cob onctueux, ourebie, phacochère, potamochère ; pour les immatures : chacal, panthère, serval, mangouste ichneumon, mangouste à queue blanche, hérisson, rat palmiste, aulacode, lièvre. Les oiseaux et reptiles suivants n'hébergeaient que des immatures : francolin, pintade sauvage, poulet, petitè outarde, vautour, hibou, coucou de pagode, pipit à dos roux; gueule tapée, vipère heurtante, caméléon.

Ce doit être originairement une tique des bovidés sauvages áfricains.

\section{DISTRIBUTION}

Amblyomma variegatum est répandu dans toute l'Afrique au sud du Sahara, sauf l'Union Sud Africaine et la South-West Africa. Il existe en Arabie, à Madasgacar, aux Comores, à la Réunion, à l'lle Maurice et a été importé aux Antilles.

DISTRIBution de Amblyomma variegalum en Afrique Occidentale.

\section{Cote d'Ivoire :}

Abidjan : mouton ; Bingerville ; Adiopodoumé : chien $(n)$; Tiassalé (IPP) ; Divo; Toumodi (IPP) ; Bondoukou (IPP) ; Ouango : chien ( $n$ ) (Bondoukou); Touba ; Séguéla ; Mankono;
Odienné ; Kabala, Kamasso, Mahandianarba, Tiemba (Odienné); Tonhoulé, Minankro: bovins, francolin ( $n$ ) (Bouaké) ; Bouaké ; bovins, chien ; Afankaha, Kationou, Ndana, Niandièplé. kaha, Niénakaha, Yéholo (Katiola); Tafiré ; Ferkes sédougou ; Peyagaravogo (Ferk.) ; Korhogo : bovins, chien.

\section{Dahomey :}

Cotonou : bovins, mouton, porc ; Agblangandan (Cotonou) ; Porto Novo ; Atchoukpa, Sakété, Sémé (Porto Novo) ; Ouidah : Oumako : aulacode, Pahou (Ouidah) ; Hountagbé (Athiémé) ; Abomey ; Agbangnisoun, Catéou, Djidja : chien, Honhoun (Abomey); Dan (Bohicon) ; Agouagon : milan ( $n$ : IPP), bovins; Paouignan, Gomé, Aligbokoto, Gbaffo, Léma, Loulé (Dassa); Dassa Zoumé ; Logozohé : bovins, lièvre (n), Gnoukpuignon, Gobada, Lahotan, Lozin, Monkpa, Ouédémé, Sozoumé (Savalou) ; Savalou ; Cabolé, Djalloukou (Tchetti) ; Savé ; Kilibo ; Kokoro, Ouessé, Toui (Kilibo) ; Boroninga (Tchaourou) ; Parakou : bovins, chat $(l)$, chien $(l)$, poule $(n)$; Ferme Okpara ; Abakourou, Baléyarou, Bapérou, Fiạrou, Ganou, Guéma, Kika, Kratébou, Yérémarou (Parakou); Nikki : bovins, mouton, Biro, Bouca, Koni, Kalalé, Bessassi (Nikki) ; Bembéréké ; Fo-Bourré, Ouari, Pessara, Sékéré, Sikki, Sokka, Camia, Ouararou, Zansarou (Bembéréké) ; Ina ; Goua (Ina) ; Bori : buffle bubale ichneumon ( $n$ ) (Ndali); Béroubouay; Frékon, Péhéra (Bérouhouay) ; Djougou : bovins, mouton; Birni : francolin ( $n$ : IPP) ; Kouandé ; Natitingou ; Koutangou, Takrissari, Yergatamou, (Natitingou) ; Tanguiéta : bovins, cheval ; Gnatokandi, Gouandé, Koutari (Tanguiéta) ; Kandi ; Gogonou : bovins, mouton, Kofo, Sondo, Toui (Kandi) ; Segbana ; Libanté, Liboussou, Piami, Sokotindji, Vela (Segbana); Séfou : bubale, hippotrague; Banikoara : mouton; Atabénou, Bahouamou, Soroko, Toussou (Banıkoara) ; Malanville; Paratégui (Guéné).

\section{Guinée Française :}

Dubréka ; Kindia ; Pastoria ; Télimélé : bovins, chien $(n)$; Barkéré, Kounna ('Télimélé) ; Pita ; Mamou ; Timbo (MHNP) ; Gada-Oundou (Dalaba) ; Labé ; Sarékali : guib (Popodara) ; Koin, Kollangui (Tougué) ; Dembo, Donta, Sabéré, Soundougou, Tounti (Diari); Daporo, Hénéré, Manassigui, Mangakouloum, Toougo (Lélouma) ; Gaoual ; Gada-Bendoun, Kalya-Maoundé, Yilmé-Roundé (Gaoual) ; Binani ; Goungouroun, Lambawol (Binani) ; Sabéréali (Mali) ; Dankama, Fouga, Koumbia (Dinguiraye); Sa- 
guiri ; Gbenkorokoro, Kiniéran, Limbana, Nounkounkan, Oudoumakoro (Siguiri); Kouroussa : bovins, chien; Sareya ; Bissikrima ; Dabola ; Bankon, Bolombaya, Dalakoundian, Daramela, Daroussalam, Donga, Douabo, Fadougou, Kalon, Kodin, Koronkonya, Kousondougou, Lilinko, Mareya, Metta, Nargonna, Thiankaye (Dabola); Farana; Bambaya : cheval, Bindougou, Koudébou, Dalafilani, Manya, Passaya, Farékoto (Farana); Kankan : homme (Joyeux, 1915), bovins; Bordo, Dalala (Kankan) ; Bélissa, Dioulabougou (Kissidoujou) ; Kongodou (Gueckédou) ; Dabadougou, Diaboìdou, Féregbéla, Gbakédou Sogboro (Beyla) ; Gogota, Kokota (Nzérékoré) ; Gouécké (MHNP).

\section{Haute Volta :}

Djigoué, Yérifoula (SGHMP), Niobini, Zinka : mouton, (Gaoua) ; Banfora (SGHMP) ; Bamako, Daramandougou, Lémouroudougou, Mangodaia ; bovin, chien $(n)$, Nafona Sitiéna : cheval, Tengréla : bovins, chien ( $n$ ), Tiéfora : bovins, chevre, Yorkofesso (Banfora); Toussiana; BoboDioulasso : bovins, cheval, âne, homme ( $n$ : SGHMP), francolin ( $n$ : SGHMP), pintade ( $n$ : SGHMP), varan ( $n$ : SGHMP), caméléon ( $n$ : SGHMP); Banankélédaga : Nianaba, Tiéra (SGHMP) (Bobo); Samandéni ; Boni (SGHMP), Dougoumato : sylvicapre (SCHMP) (Houndé); Diébougou : ourébie (SGHMP), hippotrague (SGHMP) ; Tiogo : chien (SGHMP) (Koudougou); Niassan : cheval (SGHMP) (Tougan); Léo; Ouagadougou : bovins, moutons. chève, petite outarde $(n)$; Cissin, Kampéla, Zamouna, Ziou (Ouagadougou) ; Dargo, Tengo, Zambanga (Kaya); Garango, Bakouré (Tenkodogo) ; Fada-Ngourma ; Kikidéni (Fada) ; Manga (Neumann, in tab.).

\section{Mauritanie :} so).

Diarat : zébu, mouton, Mbel, Tengoumit (Ros-

$$
\text { Niger : }
$$

Dakoro ; Zinder.

\section{Sénégal :}

Dakar : chacal (n), hérisson (n), mangouste à queue blanche (n) (Villiers, 1955); Dakar : enfant; Mbao : écureuil fouisseur (n), vautour (n) (Villiers, 1955); Ngor; Tiaroye (Dakar); Rufisque $(\mathrm{CNm})$; Sangalcam : bovins, chien, Gorom : coucou de pagode $(n)$, pie piac-piac $(n)$ (Rufisque); Lac Tamna : coucou de pagode $(n)$ (IFAN); lac Mbaouar : pipit à dos roux $(n$ :
IFAN) ; Sébikotane : vipère heurtante ( $\pi:$ IFAN), serval ( $n$ : Villiers, 1955); Keur Dembakélé.: cheval (SGHMP), Sarhor : cheval, Diassane chacal (Thiès); Dahra ; Saint Louis (CNm) ; Fatick ; Nakha Mayc, Séssène (Fatick); Kaolack; Barkayel, Bouldiabé, Diokhoul, Kaville, Keur Bouki, Koutal, Mbadio, Ndiobène, Ndoffane, Nyoth, Ouyal Sandé, Sobé, Tickat (Kaolack); Nioro du Rip ; Birkelane : chien (n) ; Diamal, Hamdallaye, Ségré (Birkelane) ; Kaffrine ; Lama Fara, Lonkane, (Kaffrine) ; Nganda ; Mbayène (Koungheul) ; Niokolo-Koba : guib, ichneumon $(n)$, mangouste à queue blanche $(n)$, potamochère (n), bubale (n), ourébie (n), hibou (n), vautour (n) (Morel, 1956), phacochère (n), céphalophe roux $(n)$; Fatick : oie de Gambie $(n)$; Kédougou : riv. Falémé : guib $(n)$, phacochère $(n)(100 \mathrm{~km}$ de Kédougou); Doubirou, Sambatara, Saré Sori, Simbi (Vélingara); Boguel, Parégardy (Kolda); Boudhié (Sédhiou) ; Bignona : guib ( $n$ : Villiers, 1955) ; Ziguinchor.

\section{Soudan Français :}

Nioro : zćbus, moutons, chacal (n) ; Bamako : animaux domestiques (Rousselot, 1951 et 1953), zébus ; Sotuba : bovins (Derbal et Balis, 1950), zébus, bovins, homme $(n)$; Kaféla, Zégoua (Sikasso) ; Koulikoro : pintade ( $n$ : MHNP); Baguineda (Girard et Rousselot, 1945) ; Ségou : animaux domestiques, hérisson, mangouste à queue blanche (Rousselot, 1951 et 1953), zébus ; Dongoufé, Fambougou, Kaba, Somon, Sotabougou, Zangon Ouéré (Ségou) ; Dioro : zébus, petite outarde (n) ; Banamba (IPP) ; Tamani ; Bani; San ; Gioutiou (San) ; Togo (IPP); Amanongo, Komba Saré, Oualo (Macina); Gouéré (Bai) (SGHMP) ; Gniminiama ; Sansa (Gniminiama); Amba : chacal (n) (Boré);

\section{Togo :}

Lomé : moutons (Ziemann, 1905) ; Agouévé, Sévagan (Lomé) ; Abobo, Avéta, Djagblé (Tsévié) ; Misahohe (Ncumann, in tab) ; Bismarckburg (Neumann, in tab) ; Pagouda ; Amaidé, Bafilo, Dako, Didaouré, Douwa, Inousayo, Koumondé, Kri-Kri, Na, Pagalam, Soudou, Tchamba, Tchavadé, Téméliwé (Sokodé); Kabou, Koundoun, Nababoun, Santé, Tchoticou (Bassari); Alloum, Birou, Dakoka, Lingaou (Niamtougou); Nataré, Tamantougou (Nakiest); Mango (Kandé) ; Dapango ; Cinkassé, Korbougou, Kpougou, Nadingou, Nanergou, Nassablé, Sanfatouti, Soumpiougou, Tantoga, Toaga (Dapango). 


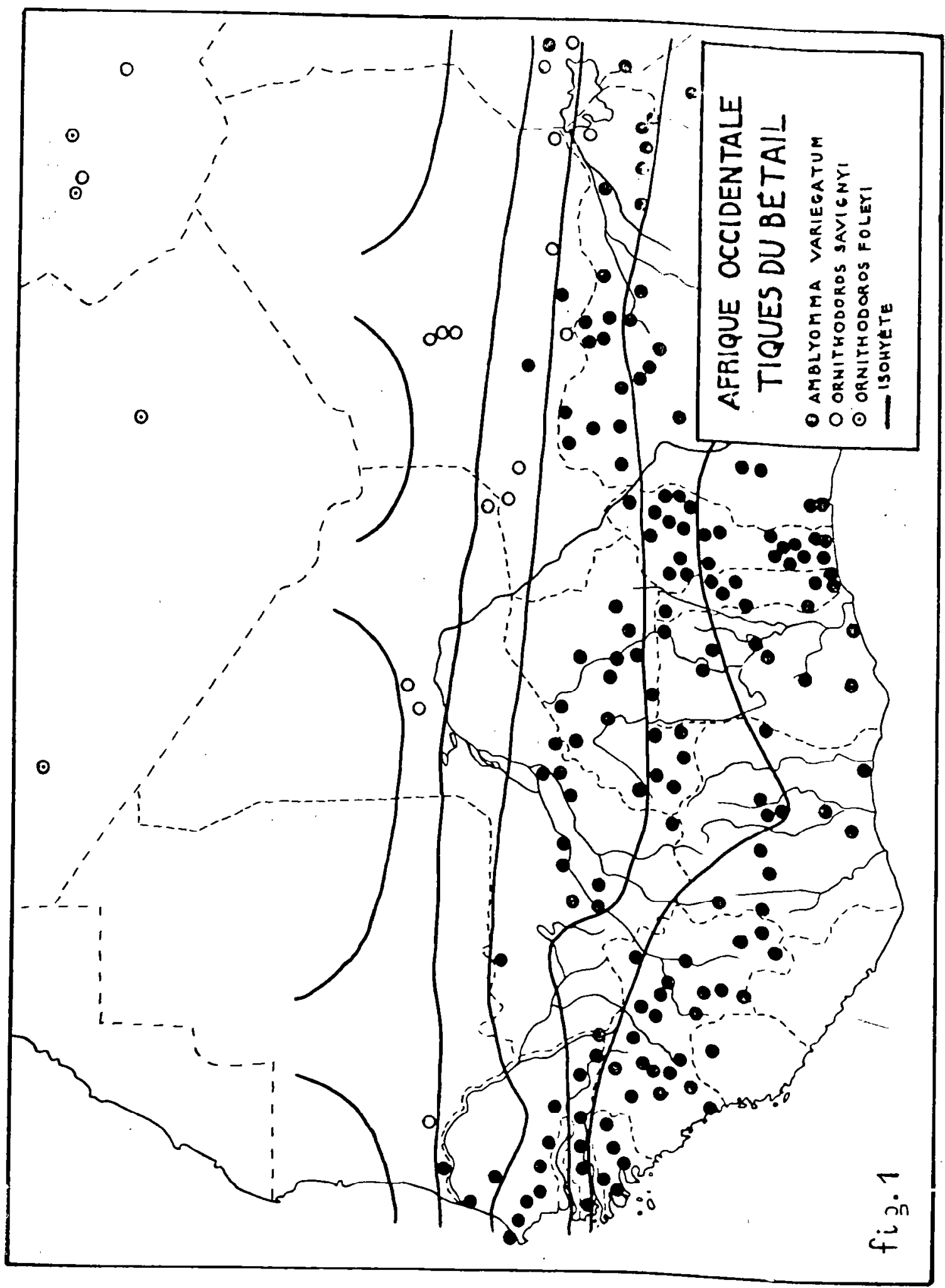




\section{Gambie :}

Bakau : cheval (Simpson, 1911).

\section{Guinée Portugaise :}

Tendeiro (1948, 1951, 1952) : Pessuba, Teixeira Pinto (Cacheu), Mansaba (bovins, moutons, chèures, cheval, âne dans ces trois localités); Pessuba : poulet (n) ; Bajucunda : francolin $(n)$; Buruntuma : pintade $(n, l)$; Buba : cob . onctueux $(n)$; Cacheu : panthère; Iles Bijagos (Tendeiro, 1953); Bissau : homme $(l$ : Villiers, 1955) ; Bafata : homme ( $:$ Villiers, 1955).

\section{Sierra Leone :}

Konta (Simpson, 1913) ; Rowerre (Simpson, 1913); Batkanu (Yorke et Blacklock, 1915); Kaballa.

\section{Gold Coast-Ghana :}

Kumasi (Simpson, 1914) ; Obuasi (Neumann, in tab.); Kona (Manpong) (Simpson, 1914); Zantana : bubale (Simpson, 1914); Wandara (Simpson, 1914) ; Larabanga : hippotrague (Simpson, 1918) ; Accra (Macfie, 1915) Bawku (Moody, 1922) ; Tamale (Moody, 1922).

\section{Nigéria:}

Nombreuses références dans la publication de Unsworth, (1952). Lokoja, Zungeru : chien, Baeri : cheval, Mina, Ere : chien (Badadri). Opelifa, Lagos (Simpson, 1911-1912); Lagos (Ziemann, 1905).

\section{Iles du Cap Vert :}

Tendeiro (1954); Sâo Vicente (Neumann, in tab.).

\section{Tchad :}

\section{Ngouri (MHNP).}

\section{0) Amblyomma paulopunctatum ( $\mathrm{Neu}^{-}$ mann, 1899).}

Tique spécifique des potamochères. On ne la trouve qu'en forêt et en régions de forte pluviosité (au-dessus de $1500 \mathrm{~mm}$ annuels). Tendeiro (1951) la signale sur porc aux lles Bijagos, Rageau (1953), sur zébu et porc à Yaoundé: Les cas de parasitisme d'animaux domestiques sont très peu souvent signalés. Pour l'Afrique Occidentale l'espèce est connue de Conakry (Neumann, 1897); Robertspoort (Neumann, 1908) ; Mansaba, Cacheu (Tendeiro, 1948, 1951, 1952) ; Bewama (Robinson, 1926 :
Sierra Leone) ; nos références concernent Yapo : homme, et Dabou : porc (Côte d'Ivoire).

30) Amblyomma splendidum Giebel, 1877.

Tique spécifique du buffle dans la sousrégion occidentale d'Afrique, elle $\mathrm{y}$ est parfois signalée comme parasitant le bétail : Yola (Unsworth, 1952 : Nigeria); diverses localités du Cameroun où, d'après l'auteur, elle est assez fréquente sur les bovins (Rageau, 1951, 1953); nous l'avons reçue de Kaballa (Sierra Leone) sur bovin.

L'cspèce est présente en Guinée Portugaise (Tendeiro, 1948, 1951, 1952 : Fulacunda, Buba), Sierra Leone (Simpson, 1911 : Yiraia ; Robinson, 1926 : Medina, Koinadugu, Firawa, Kabuga, Fulakunko), Liberia (Bequaert, 1930 : Miamu), Côte d'Ivoire (Rousselot, 1951, 1953 et Villiers, 1955 : Bouaflé), Gold Coast-Chana (Simpson, $1914=$ Robinson, 1926 : Yeji), Nigeria (Robinson, 1926 : Tegi, Lagos). Nous pouvons ajouter les références suivantes : Kete Krachi (Cold Coast : $\mathrm{CNm}$ ), rivière Zio (à l'est du Mont Agou (Togo) : Neumann, in tab.), Bori (Dahomey) : buffle, Kindia (IPP) : buffle.

\section{0) Amblyomma nuttalli (Dönitz, 1909.)}

La seule référence de l'espèce que nous possédions sur un animal domestique, en A.O.F., est Parakou : chat $(n, l)$. C'est un parasite des varans et tortues; les immatures peuvent se rencontrer sur les mammifères et oiseaux (hérisson, ichneumon, homme, coucou de pagode, francolin); c'est une espèce de savane humide et de forêt.

Depuis la rédaction de cette note 3 nymphes ont été récoltées sur bovin à Gbaffo (Dassa Zoumé : Dahomey).

\section{$\left.5^{\circ}\right)$ Boophilus decoloratus (Koch, 1844).}

\section{BIOLOGIE}

Comme toutes les espèces du genre, $B$. decoloratus est une tique à un seul hôte : la larve après son repas mue sur place, sans se retirer du point de fixation; après sortie de la nymphe, la dépouille larvaire demeure un certain temps encore implantée; la nymphe se gorge sur le même hôte, et mue de même que la larve; les adultes qui éclosent effectuent leur repas sur ce même hôte. Ainsi au cours du cycle il n'y a eu aucune phases libres sur le sol ou dans les herbes entre les repas. Les Boophilus sont affiliés aux rumi- 
nants, et peut-être aux bovins d'une façon primitive.

Le cycle d'évolution d'un Boophilus peut donc se passer en un temps beaucoup plus ramassé que chez une espèce d'un autre genre, qui demeurera quelque temps libre entre chaque repas. On peut estimer que pour un Boophilus le cycle s'effectue en un mois sur l'hôte, c'est-àdire de la fixation de la larve jusqu’à la chute de la feme'le gorgée. Il faut compter encore le temps d'oviposition et d'évolution des cufs, soit 6-7 semaines, ce qui porte le cycle complet de 2 mois et demi à 3 mois. D'après Lounsbury (1905), Theiler, A. (1911), Lewis (1939), le cycle de la femelle sur l'hôte demande de 3 semaines à 1 mois, l'oviposition 6-9 jours et l'éclosion des larves 5 semaines. Les mâles demeurent plus longtemps sur l'hôte.

En raison de ce cycle court il y a plusieurs générations par an : en fait, on trouve $B$. decoloratus presque toute l'année, quoiqu'il soit de beaucoup plus abondant en saison d'hivernage. C'est alors numériquement la deuxième tique après $A$. variegatum, dans les régions où ils sont communs tous deux.

Les femelles n'ont pas de lieux de fixation définis : fanon, ars, mamelles, etc: aussi bien quencolure, garrot, région lombaire ou chignon. Les mâles et immatures se fixent également partout. Du fait de leur petite taille, ils sont rarement prélevés dans les prospections courantes. Personnellement nous les recherchons vers la pointe des oreilles (face interne), dans la région de l'encolure, de l'épaule et du garrot ; chez les petits ruminants il faut examiner les oreilles, la tête, le périnée et entre les doigts.

L'absence de phase libre entre les repas, qui élimine les hasards qui font que chez les autres espèces une partie seulement des nymphes et adultes retrouve à se fixer, assure au contraire dans le cas des Boophilus la survie de la presque totalité des individus de chaque stade, jusqu'à la ponte. Ici la recherche d'un hôte n intervient qu une fois, non trois. Tout ceci explique le grand pouvoir de maintien de cette espèce dans une région donnée.

Cette situation a cependant sa contre-partie, car dans le cas de traitements systématiques du bétail on est assuré de toucher un plus grand nombre d'individus quand il s'agit de Boophilus plutôt que d'une autre espèce : on agit beaucoup plus efficacement puisque sur 3 stades à la fois (par exemple chez Hyalomma truncatum et Rhipicephalus simus on ne peut espérer atteindre les immatures, qui évoluent sur rongeurs sauvages et oiseaux). Ainsi malgré tout Boophilus decoloratus est une espèce qui, quoique redoutable par son abondance et le pouvoir vecteur des piroplasmoses du bétail, est relativement facile à maitriser, quand des résistances aux insecticides n"interviennent pas.

\section{HYGROPHILIE}

En A.O.F., Boophilus decoloratus apparait aux environs de l'isohyète des $500 \mathrm{~mm}$, devient abondant aux environs des $1000 \mathrm{~mm}$ jusquen forêt. Ceci concorde avec les résultats de $\mathrm{G}$. Theiler (1949) en Afrique du Sud, où 15 inches (environ $525 \mathrm{~mm}$ ) de pluies annuelles représentent le niveau critique compatible avec le maintien du Bonphilus. Sur la carte de répartition l'auteur indique des exemplaires dans des zones à moins de 15 inches (de 5 à 10 inches), mais il doit s'agir d'une introduction dans des régions où des conditions d'humidité locale entretiennent le Boophilus sur une aire restreinte.

\section{HOTES}

Bovins et zébus sont infestés souvent par un grand nombre de tiques; les petits ruminants, infestés moins souvent, le sont seulement par quelques-unes. Le cheval et l'âne sont fréquemment parasités. Les animaux sauvages sont rarement porteurs de la tique, et de quelques exemplaires seulement : guib, bubale, hippotrague, gazelle, buffle, phacochère. La mention sur pintade (Haute Volta) est inattendue.

\section{DISTRIBUTION}

L'espèce est présente dans toute l'Afrique au Sud du Sahara. A Madagascar elle est remplacée par B. fallax Minning $=$ microplus, introduite avec le bétail d'Asie tropicale (Buck, 1948 ; Hoogstraal, 1956).

Distribution de Boophilus decoloratus en Afrique occidentale :

\section{Côte d'lvoire :}

Abidjan : bovins, chèure ; Adiopodoumé : chien; Bingerville; Divo; Toumodi (IPP); Soko (Bondoukou) ; Touba ; Séguéla : Mankono ; Kabala, Kamasso, Mahandianarba, Tiemba (Odienné) ; Tonhoulé, Minankro (Bouaké) ; Afankaha, Kationou, Ndana, Niandiéplékaha, Niénakaha, Yékolo (Katiola) ; Korhogo : bovins, chien.

\section{Dahomey :}

Cotonou ; Agblangandan (Cotonou) ; Sakété, 


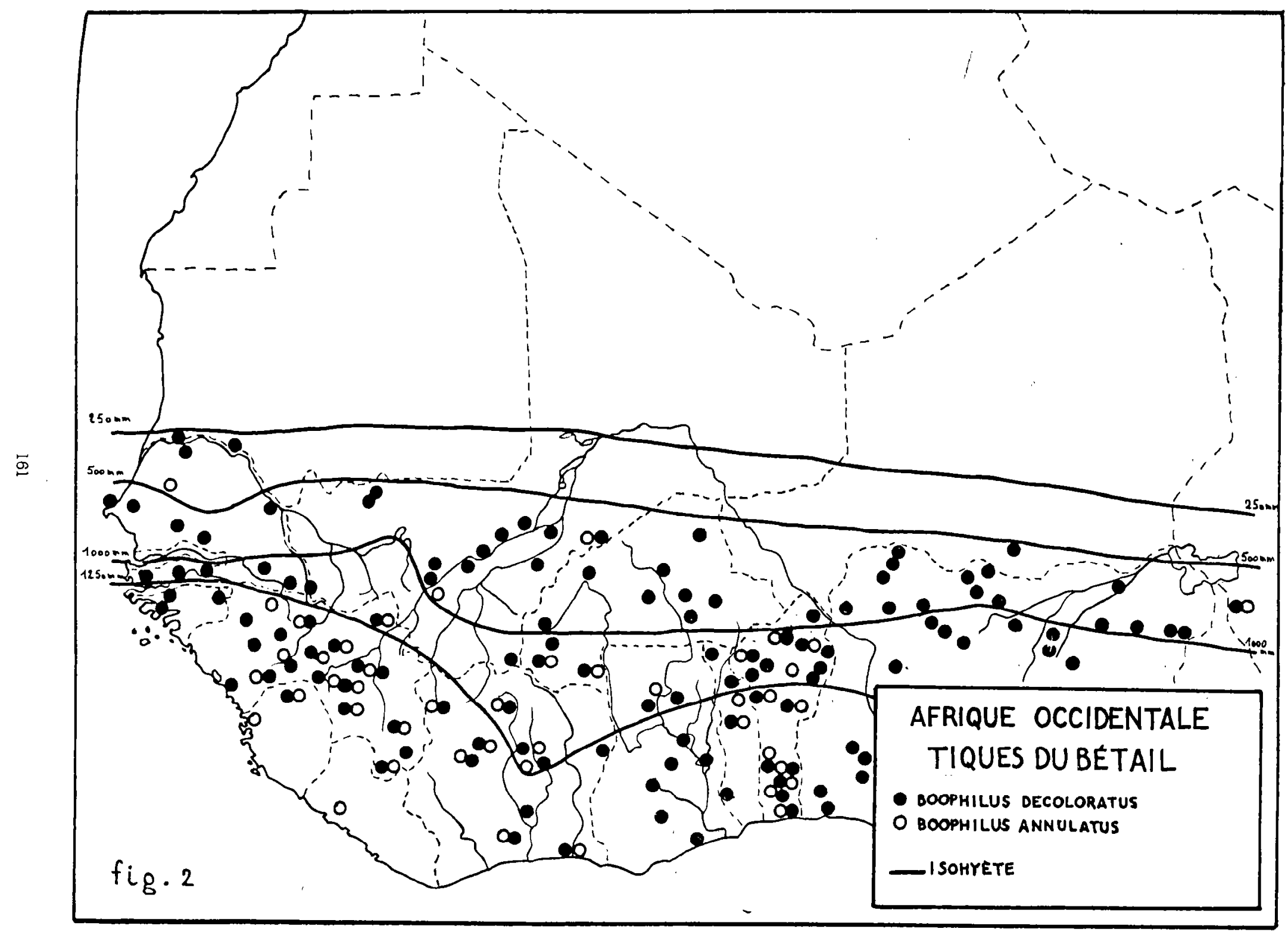


Sémé (Porto Novo) : Djidja : chien (Abomey); Dan (Bohicon) ; Agouagon ; Paouignan, Cbaffo, Léma, Gomé, (Dassa Zoumé) ; Dassa Zoumé ; Logozohé, Gnoukpuignon, Gobada, Lahotan, Lozin, Monkpa, Sozoumé (Savalou); Savalou ; Djalloukou (Tchetti) ; Toui (Kilibo) ; Parakou ; Ferme Okpara : Ganou, Guéma, Kika (Parakou); Nikki ; Biro, Koni, Kalalé, Bessassi (Nikki); Bembéréké : Sékéré, Sikki, Sokka, Gamia Zansarou (Bembéréké); Ina ; Bori : bubale (Ndali); Béroubouay; Frékou, Pébéra (Béroubouay); Djougou ; Kouandé ; Natitingou ; Takrissari (Natitingou); Tanguiéta ; Gnatokandi, Koutari (Tanguiéta) ; Kandi ; Angaradébou, Gogonou, Kofo, Tioka, Toui (Kandi) ; Libanté, Liboussou, Piami (Segbana); Bahouamou, Soroko (Banıkoara); Malanville; Karimama.

\section{Guinée Française :}

Dubréka ; Kindia ;Pastoria ; Barkéré, Kounna, Orémendou, Singuéléma (Télimélé) ; Mamou ; Timbo (MHNP) ; Gada-Oundou (Dalaba) ;Labé ; Koin (Tougué) ; Dembo, Tounti (Diari) ; Manassigui (Lélouma) ; Gaoual ; Gada Gendoun (Gaoual) ; Sabéréali (Mali) ; Dankama, Fouga, Koumbia (Dinguiraye); Siguiri ; Bamissakoro, Banfélékoro, Gbenkorokoro, Kiniéran, Nounkounkan (Siguiri); Kouroussa : bovin, chien; Sareya ; Bissikrima ; Dabola ; Baniré, Bankon, Dalakoundian, Daraméla, Daroussalam, Douabo, Fadougou, Kalon, Kodin, Konkoronya, Kousondougou, Lilinko, Mette, Nargonna, Thiarkaye (Dabola) ; Farana ; Bambaya, Bindougou, Koudébou, Dalafilani, Diana, Kobikoro, Manya, Passaya, Sarékoro (Farana) ; Kankan; Bordo, Dalala, Touba : âne (Kankan); Bélissa, Dioulabougou (Kissidougou); Korodou; Albadaria (Ouassamandou) ; Soumdou, Yrandou (Guéckédou) ; Beyla ; Balanfé, Daladougou, Diaboidou, Féregbéla, Gbakédou, Sogboro (Beyla); Gogota, Kokota (Nzérékoré) ; Gouécké (MHNP).

\section{Haute Volta :}

Djigoué, Sixlalla, Tielkan, Zinka : mouton (Gaoua) ; Banfora (SGHMP), Bossié, Daramandougou, Koindé, Kolokolo, Mondon, Moussodougou, Nafona, Tengréla, Tiéfora (Banfora); Bobo-Dioulasso : bovins, cheval ; Banankélédaga ; Samandéni ; Barani : cheval (Dédougou : SGHMP); Ouagadougou : âne; Sohotenga : pintade (Ouagadougou); Dargo, Yengo (Kaya); Batibogou, Sampelga (Dori); Békouré, Garango (Tenkodogo) ; Fada Ngourma ; Kikidéni (Fada) ; Koupéla (IPP).

\section{Mauritanie :}

Mbel (Rosso); Moundi (Boghé).

$$
\text { Niger : }
$$

Zinder.

\section{Sénégal :}

Dakar : chien (IFAN) ; Hann (Zoo) : oryx, gazelle dama, hippotrague (Dakar) ; Sangalkam (Rufisque); Keur Dembakélé : cheval (SGHMP : Thiès); Hamdallaye (Birkelane); Koungheul : Saint Louis (IPP) ; Dahra ; Dagana ; Bakel ; Niokolo-Koba : bubale (Morel, 1956); Kédougou ; rivière Falémé : guib, phacochére $(100 \mathrm{~km}$ de Kédougou) ; Kolda (IPP) ; Boudhié (Sédhiou); Ziguinchor : bovins, moutons.

\section{Soudan Français :}

Nioro : zébu, mouton, cheval, âne ; Gavignané (Nioro) ; Bamako (Rousselot, 1951, 1953), zébus ; Sotuba ; Ségou (Rousselot. 1951. 1953), moutons : Somon, Zangon Ouéré (Ségou); Dioro: Banamba (IPP) ; Tamani ; Bani ; Markala ; cheval (Villiers, 1955); Niono ; San ; Békuy (San) ; Togo (IPP); Oualo (Macina); Djenné : cheval (IPP) ; Gouéré (Bai); Gniminiama ; Sansa (Gniminiama); Amba : chacal (Boré).

\section{Togo :}

Misahôhe (Neumann, in tab.) ; Sokodé (Neumann, in tab.); Amaidé, Bafilo, Bahouda : cheval, Didaouré : mouton, cheval, Komah : cheval, Koulondé : cheval, Koumondé, Malfakassa, Na, Sondou, Soutouboua (Sokodé); Mango (Kandé) ; Korbougou, Nassablé, Todjinga (Dapango).

\section{Guinée Portugaise :}

Pessuba, Bissorà, Canchungo (Tendeiro, 1948, 1951, 1952).

Iles du Cap Vert :

Neumann (1901) ; Bacelar (1950) ; Sâo Vicente (Neumann, in tab.).

\section{Sierra Leone : \\ Kamagota (Hoogstraal, 1954); Kaballa. \\ Gold Coast-Ghana :}

Accra (Macfie, 1915) ; Makorgo (Simpson, 1914) ; Prang : cheval (Simpson, 1914) ; Mampong (Simpson, 1914); Zantara : hippotrague (Simpson, 1914); Obuasi (Neumann, in tab.); Kete Krachi (Neumann, in tab.); Tamale (Moody, 1922) ; Bawku (Moody, 1922). 


\section{Nigéria :}

Nombreuses références dans la publication de Unsworth (1952). Simpson (1912) signale l'espèce de Aro : chien, Oshogbo, Abeokuta : mouton, Opelifa : cheval ; Ziemann (1905) de Lagos : bouf, mouton.

\section{$\left.6^{\circ}\right)$ Boophilus annulatus (Say, 1821).}

Une espèce proche de $B$. annulatus a été décrite de Brazzaville sous le nom de $B$. congolensis par Minning en 1935. Elle a été retrouvée depuis au Cameroun (Rageau, 1953), au Congo Français (Rousselot, 1953), au Congo Belge (Theiler G. et Robinson B., 1954).

Hoogstraal (1954) examine dars la collection Nuttall du British Museum deux lots de Boophilus, provenant de Sierra Leone (Kamégota) et Nigéria (Obubura), qu'il détermine annulatus. En 1956 il retrouve l'espèce au Sudan et met $B$. congolensis en synonymie avec annulatus.

Nos exemplaires, rapportés d'abord à congolensis, ne peuvent pas être différenciés des annulatus d'Afrique du Nord observés dans les collections de la Faculté de Médecine, de l'Institut - Pasteur de Paris ou de la collection Neumann, à Toulouse. Nous estimons donc la synonymie légitime.

Boophilus annulatus est problablement originaire du bassin méditérranéen ou du Proche Orient. Il a dû être introduit en Amérique du Nord et sur la côte du Golfe de Guinée à partir du XVI ${ }^{\mathrm{e}}$ siècle par les transports de bétail originaire d'Espagne, Portugal ou Italie.

Hoogstraal (1956) au contraire estime que cette tique est originaire d'Amérique du Nord et parasitait primitivement les cerfs et les bisons.

Le transport à grandes distances des Boophilus est favorisé par le long séjour sur l'hôte. Aucune autre tique $n$ avait les mêmes chances de dissémination. L'extension de Rhipicephalus sanguineus et Hyalomma dromedarii ou rufipes tient à d'autres fecteurs.

\section{BIOLOGIE}

Elle est semblable à celle de $B$. decoloratus.

Les auteurs (Rageau, 1953 ; Rousselot, 1953) rapportent que l'espèce est peu abondante, quelques exemplaires se trouvant dans des lots de $B$. decoloratus. Ce n'est pas du tout le cas pour l'A.O.F. où on trouve cette tique en relative abondance. Dans certíns prélèvements nous avons rencontré l'espèce seule ou en majorité par rapport à $B$. decoloratus. En fait c'est une tique commune en région guinéenne et forestière.

\section{HYGROPHILIE}

Cette tique est beaucoup plus exigeante vis à vis de l'humidité que $B$. decoloratus. En A.O.F. elle n'apparaît qu'au sud de l'isohyète des $1000 \mathrm{~mm}$. Elle n'est donc présente qu'en zone soudanienne sud et guinéenne.

\section{DISTRIBUTION}

B. annulatus doit se trouver dans tous les territoires bordant le golfe de Guinée, jusqu'au Congo. A l'intérieur, il s'avance jusqu' au Sudan Rep. (Hoogstraal, 1956). Il est présent en Oubangui-Chari, à Bangui et Fort Sibut (IPP).

Distribution de Boophilus annulatus en Afrique Occidentale :

\section{Côte d'Ivoire :}

Bingerville ; Divo ; Séguéla ; Mankono ; Kamasso, Mahandianarba (Odienné); Tonhoulé, Minankro (Bouaké) ; Afankaha, Kationou, Ndana, Yékolo (Katiola) ; Korhogo.

\section{Dahomey :}

Cotonou ; Agblangandan (Cotonou) ; Atchoukpa, Sakété, Sémé (Porto Novo) ; Djidja : chien (Ábomey); Dassa Zoumé ; Paouignan, Gbaffo, Léma, Gomé (Dassa Zoumé) ; Logozohé, Cnoukpuignon, Gobada, Lahotan, Lozin, Monkpa, Sozoumé (Savalou); Savalou; Djalloukou (Tchetti) ; Agouagon ; Kukuro, Ouessé, Toui (Kilibo) ; Parakou ; Ferme Okpara ; Guéma (Parakou) ; Biro (Nikki) ; Sékéré (Bembéréké); Djougou ; Tanguiéta ; Kandi ; Angaradébou, Gogorou, Kofo, Tioka, Toui, (Kandi) ; Bahouamou, Soroko (Banikoara).

\section{Guinée Française :}

Dubréka ; Kindia ; Pastoria ; Mamou ; Kollangui, Koin (Tougué): Dembo (Diari) ; Hénéré, Marassigui, Toougo (Lélouma) ; Sabéréali (Mali); Siguiri; Fouga, Koumbia (Dinguiraye); Sareya ; Bissikrima ; Dabola ; Baniré, Daroussalam, Douabo, Fadougou, Kalon, Konkoronya, Nargonna (Dabola); Farana ; Birdougou, Koudébou, Dalafilani, Diana, Kobikoro (Farana); Kankan ; Dalala, Faralako (Kankan); Bélissa, Dioulebougou (Kissifougou); Balanfé, Dabadougou, Dic boidou, Férégléla (Beyla); Kokota (Nzérékoré). 


\section{Haute Volta :}

Silalla (Gaoua); Banfora ; Lémouroudougou, Nafona, Tengréla, Tiéfora, (Banfora); Toussiana.

\section{Soudan Français :}

Sotuba (présence probablement consécutive à l'introduction de bétail provenant de Côte d'Ivoire) ; Gouéré (Bai : SCHMP) (il doit s'agir d'une récolte sur du bétail en transhumance).

\section{Togo :}

Didaouré : cheval, Komah : cheval, Malfakassa, $\mathrm{Na}$ (Sokodé) ; Mango (Kandé).

\section{Sierra Leone :}

Kamagota (Hoogstraal, 1954) ; Kaballa. Simpson (1913) désigne très problabement sous le nom de $B$. australis un Boophilus différent du decoloratus, qui a toute chance d'être un annulatus. On sait en effet que Dönitz (1905) a appliqué le nom de $B$. australis Füller à des Boophilus d'Afrique voisins du $B$. annulatus nord-américain. C'est sans doute sur l'autorité de Donitz que Nuttall a nommé australis des $B$. annulatus trouvés par Simpson en Afrique Occidentale, à Konta, Laminaia et Rowerre, sur bovins. Il en est de même des exemplaires de Yorke et Blacklock (1915) de Batkanu, que Nuttall avait également déterminés de cette manière. Il subsiste toutefois un doute sur l'identité réelle de ces Boophilus.

\section{Gold Coast-Ghana :}

Même doute que pour les références précédentes. Daboya : hippotrague (Simpson, 1918 : B. australis) ; Makongo (Simpson, 1914 : B. australis).

\section{Libéria :}

Kakata (Hoogstraal, 1956).

\section{Nigéria :}

L'espèce y est problabement aussi fréquente gue dans les territoires voisins. Cependant Unsworth (1952) semble l'avoir méconnue. Hoogstraal (1954) la signale d'Obubura.

\section{$\left.7^{\circ}\right)$ Haemaphysalis aciculifer Warburton,} 1913 (=H. aciculifer rugosa T.S. Dias, 1956).

C'est une tique d'animaux sauvages (surtout des herbivores) en régions soudanienne et guinéenne, accidentellement rencontrée sur le bétail, à quelques exemplaires. Pour l'Afrique Occi- dentale les références sur bovins sont les sujvantes.

Daramandougou (Banfora : Haute Volta); Ouararou (Bembéréké), Gomé (Dassa), Parakon, Toui (Kandi) (Dahomey) ; Bauchi, Jakari, Effon (Unsworth, 1952).

Sur animaux sauvages l'espèce est signalée des localités suivantes : Bobo-Dioulasso, Dougoumato (Houndé), Diébougou (Haute Volta); Toumodi (Côte d'I voire) ; Niokolo-Koba (Morel, 1956, Sénégal) ; Wandara (Simpson, 1913 ; Nuttall et Warburton, 1915) (Gold Coast).

$\left.8^{\circ}\right)$ Haemaphysalis hoodi Warburton et Nuttall, 1909.

C'est une tique spécifique des oiseaux, à tous les stades. Ses hôtes sauvages les plus fréquents sont les coucous de pagode et les francolins. Elle est parfois signalée sur le poulet. Ce parasitisme peut prendre des formes très graves (Lucas, 1954) Nos références sur poulets sont les suivantes : Bathurst (Warburton et Nuttall, 1909 : Gambie) ; Kolda (IPP : Sénégal).

Nous possédons la tique de Sangalkam, Gorom, Mbour, Messira du Niombato, Kaffrine (Sénégal); Sotuba (Soudan); Bouaké, Divo, Tafiré (Côte d'Ivoire) ; Kopargo, Bassila, Parakou, Atchéribé (Dahomey); Bobo-Dioulasso (SGHMP : Haute Volta).

$\left.9^{\circ}\right)$ Haemaphysalis leachi leachi (Audouin, 1827).

Cette tique de carnivores sauvages parasite le chien et le chat plus ou moins fréquemment. En raison de cette particularité, c'est dans une publication sur les tiques d'animaux sauvages d'A.O.F. que nous traiterons plus complètement de cette espèce. Elle est répandue sous diverses formes en Afrique Ethiopienne et en Asie Tropicale.

Le parasitisme des chiens par $H$. leachi est moins important en Afrique occidentale (où le chien présente surtout Rhipicephalus sanguineus) qu'en d'autres régions ('Afrique du Sud, par exemple). Le cycle s'effectue sur trois hôtes. Les immatures se gorgent sur rongeurs, insectivores, etc...

A partir d'un minimum de $500 \mathrm{~mm}$ de pluies annuelles, $H$. leachi ne semble pas avoir d'autres exigences à ce sujet, car on la trouve au sud du Sahel jusqu'en forêt. 
Dans les références qui suivent, nous avons précisé l'hôte seulement quand il s'agit d'un animal domestique.

\section{Côte d'lvoire :}

Adiopodoumé (Abidjan) ; Bondoukou (IPP) ; Korhogo : chien.

\section{Dahomey :}

Pobé : chien, Tohoué : chien (SGHMP), Sakété : chien (Porto Novo) ; Agouagon : chien (IPP) ; Parakou : chat.

\section{Guinée Française :}

Labé : chien (SGHMP) ; Télimélé : chien ; Dabola (IFAN) ; Friguiagbé (MHNP) ; Mt Nimba (Villiers, 1955).

\section{Haute Volta :}

Santidougou,: (SGHMP), Pama (SGHMP) (Bobo) : Batié (SGHMP).

\section{Sénégal :}

Hann : chat (Dakar) ; Sangalcam : chat (Rufisque); Tiaroya (Villiers, 1955) ; Richard Toll (IFAN) ; Nayes (IFAN); Nioro du Rip (IFAN); Messira du Niombato (IFAN) ; Bignona (Villiers, 1955) ; Tambacounda (IPP) ; Niokolo-Koba (Morel, 1956) ; Poperguire : chat ; Sandiara (Mbour).

\section{Soudan Français :}

Nioro : chat ; Lorak Banr (Nioro) ; Kayes (IFAN) ; Sikasso ; Gogoro (Douentza) ; Bamako, Ségou (Rousselot, 1951, 1953).

\section{Togo :}

Lomé ; Misahòhe : chat (CNm) ; Bismarckburg (Neumann, in tab.).

\section{Sierra Leone :}

Nombreuses localités, citées par Simpson (1913) et Nuttall et Warburton (1915) : chiens, bovins, moutons, chévres et hôtes sauvages.

\section{Gold Coast-Ghana}

Nombreuses localités dans Simpson (1914), Nuttall et Warburton (1915) : chien, cheval, bovins. (Kumasi, Prang, Makongo, Jattos-Zonga, Adda, Accra) et Moody (1922) : Accra, Kumasi, Tamale.

\section{Guinée Portugaise :}

Buruntuma, Cussara, Buba (Tendeiro, 1948, 1951. 1952) : chien et animaux sauvages.
Nigéria :

Nombreuses localités dans Simpson (1912), Nuttall et Warburton (1915) : chiens, moutons, animaux sauvages.

\section{0 $0^{\circ}$ Haemaphysalis parmata (Neumann, 1905).}

Comme $H$. aciculifer, c'est une tique d'animaux sauvages qui se retrouve plus ou moins fréquemment sur animaux domestiques. Son domaine est la région guinéenne, surtout en forêt.

Nous n'avons pas de référence.de l'espèce sur le bétail en A.O.F. Simpson (1913) la signale de Securella : bovins (Sierra Leone), Ziemann (1905) de Lagos : bovins.

En A.O.F. elle est présente à Yapo (Villiers, 1955), Tai, Nzida, Divo, Koléahinou (Soubré), Adiopodoumé, Dabou, Toumodi (IPP), Tiassalé, Zaranou (IPP), Banvayo (SGHMP : Bouna) (Côte d'Ivoire) Atchéribé (IPP : Dahomey).

110) Hyalomma dromedarii, Koch, 1844.

\section{BIOLOGIE}

Le nombre d'hôtes de cette tique est mal défni. En fait il doit être variable et dépendre des souches et des corditicns climatiques. Delpy ét Gouchey (1937) la considèrent comme une tique à trois hôtes, qui peut n'en utiliser que deux si les conditions deviennent défavorables. En Iran le cycle le plus court a demandé 93 jours en saison chaude. En saison froide il faut plus de 7 mois (280 jours). Il y a deux générations par an.

Pour Hoogstraal (1956) en Egypte, H. dromedarii effectue son cycle sur 2 hôtes $\left(1^{\circ}\right.$ larvenymphe, $2^{\circ}$ adulte).

En A.O.F. on trouve des adultes toute l'année.

\section{HYGROPHILIE}

Les Hyalomma s'accommodent d'une faible moyenne annuelle de pluies. Bien plus, ils ne peuvent subsister dans la plupart des cas, lorsque cette moyenne s'élève trop. Ce sont, avec certains Ornithodoros, les plus xérophiles des lxodoïdes.

L'adaptation extrême est le fait de $H$. dromedarii, qui en A.O.F. ne semble pas dépasser au sud l'isohyète des $500 \mathrm{~mm}$ et re doit pas se reproduire par moins de $100 \mathrm{~mm}$ annuels. C'est donc une espèce subdésertique. L'aire ainsi 
délimitée s'accorde avec l'aire de reproduction et de vie normale des Camélidés.

\section{HOTES}

L'hôte originel des adultes est le chameau (ou le dromadaire). Véhiculée par lui, cette tique s'adapte aux autres herbivores domestiques sur toute son aire de répartition.

Les immatures sont surtout rencontrés sur le chameau. En Egypte et au Yemen Hoogstraal (1956) les trouve sur lièvres, gerbilles, gerboises, psammomys, 'merions, hérissons.

En A.O.F. nous avons rccucilli les adultes sur dromadaire, zébu, cheval, oryx, mouflon, phacochère. Sur hérisson, chien et lièvre rous avons trouvé des immaturcs qui nc pcuvent être déterminés exactement (matériel reçu mort). Il s'agit de $H$. dromedarii ou de $H$. impeltatum.

\section{DISTRIBUTION}

L'espèce est répandue partout où il $\mathrm{y}$ a des chameaux : Tibet, Mongolie, Afghanistan, Pakistan, Russie, Proche-Orient, Afrique du Nord. $\mathrm{Au}$ sud du Sahara elle existe, en dehors de l'A.O.F., au Soudan, en Ethiopie, en Somalie (française, italienne, britannique) et peut-être au Kenya (Hoogstraal, 1956). Elle est présente au Tchad à Ati (CIPP).

\section{DISTRIBUTION DE Hyalomma dromedarii EN AFRI- QUE OCCIDENTALE}

Dans le cas de cette espèce l'hôte n'est pas spécifié lorsqu'il s'agit du dromadaire, ou que les exemplaires ont été trouvés au sol.

\section{Mauritanie :}

Kedia d'Idjil (Villiers, 1955); Atar (Villiers, 1955) ; Molomhar (Atar) (Villiers, 1955) ; Hassi cl Motlch (Villiers, 1955); Akjoujt ; Moudjour (Akjoujt); Boutilimit ; Aleg; Mokhtar el Ajar (Aleg); Moudjeria ; dromadaire, zébu ; Tamchaket ; Togba (Aioun) : zébus ; Aioun el Atrous ; probablement Port Etienne (Nuttall 1925 p.p.); peut-être Atar : hérisson ( $n:$ ou $H$. impeliatum).

\section{Soudan Français :}

Nioro : zébu ; Niafunké : phacochère (MHNP) ; Tombouctou (IPP) ; Gao (IPP) : zébus ; Agamor (MHNP) ; Tadaq : oryx, dromadaires (MHNP), In Ouri (MHNP) (Adrar des Ifoghas).

\section{Sénégal :}

Mbétété (Kébémer) : Louga : cheval.

\section{Haute Volta :}

Tin Akof.

\section{Niger :}

Dakoro : dromadaire, cheval ; Agadès : dromadaire, chien ( $n:$ ou $H$. impeltatum); Anou Araren : dromadaires, zébus, Marandet, In Gall : lièvre $(n:$ ou H. impeliatum) ; Bou Gouten : dromadaire, cheval, (Agadès); Iférouane (MHNP); In Azaoua (MHNP).

\section{Sahara :}

Tin Aberda : mouflon (MHNP); Timissao (MHNP) ; Abalessa (Tamanrasset) : hérisson ( $n, l:$ ou $H$. impeltatum); In Salah (CBpt); Hassi el Khénig, oued Tinikert (Foley, 1929); Temassinin (MHNP); oued Isséyen, oued Tilillin, oued Issandilen, Adjiri, Teini, oued Tanar, Tin Taradjéli, Ranklit (Tassili des Ajjer : Colas-Belcour et Jacquemin, 1953).

$$
\text { Lybie (Fezzan) : }
$$

Brak, Ghat, Traghen, Ubari (Tonelli-Rondelli, 1932) ; Murzuk, Tmessa, Uenzerich (TonelliRondelli, 1935); Edri, Sebha, Sokna (Garibaldi, 1935) ; Bir Abaneur, Ghat (Colas-Belcour et Jacquemin, 1953).

\section{2 ) Hyalomma excavatum Koch, 1844.}

Cette espèce n'a pas encore été trouvée en A.O.F., mais il y a toute chance pour qu'elle soit présente au nord de la Mauritanie et du Niger. Elle est présente dans le Sud algérien, à proximité de la frontière du Niger : oued Isséyen, Adjiri, Tazroukou, Timaksiouine, Tementa, Tin Taradjeli, Fort Gardel, Aharar (Tassili des Ajjer : Colas-Belcour et Jacquemin, 1953) ; oued Issandilen, Teini (IPP $=H$. dromcdarii p.p. de Colas-Belcour et Jacquemin, 1953). Ces auteurs avaient aussi nommé $H$. savigniyi $=$ marginatum de quelques localités. $C_{c}$ sont des femelles. Nous les avons examinées : leur appartenance à l'espèce marginatum Koch est douteuse. De plus cette localisation s'accord it múl avec ce que l'on sait de la biologie de H. marginatum, qui supporte plus difficilement que $H$. excavatum les climats très secs.

Hoogstraal (1956) signale une forme proche

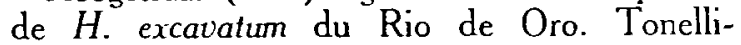
Rondelli en a décrit deux formes synonymes : $H$. fezzanensis d'Ubari, et $H$. zavattarii de Murzuk (1935). 
Retour au menu

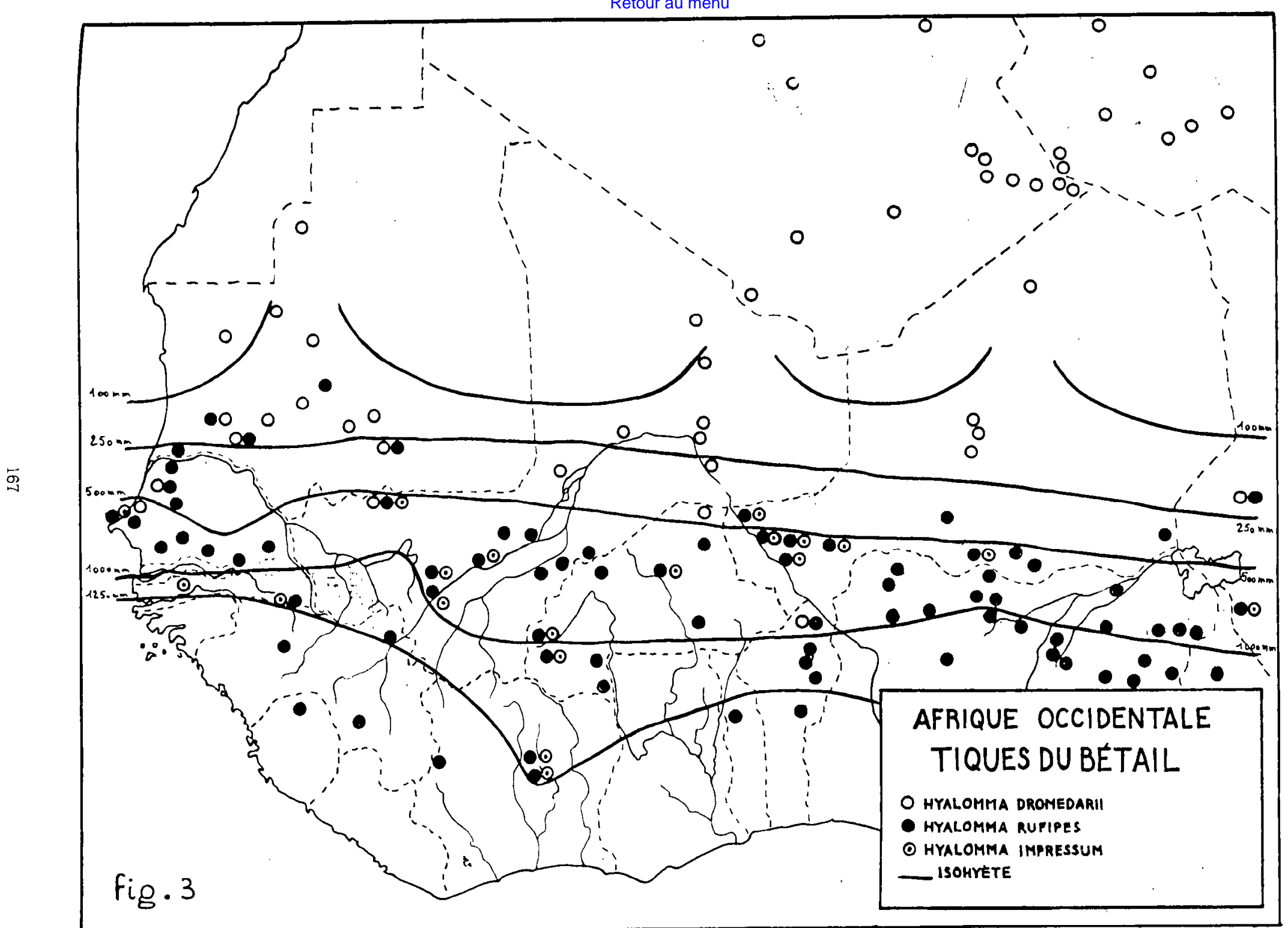


130) Hyalomma impeltatum Schulze et Schlottkc, 1930 ; Kratz, 1940.

$$
\text { (= H. brumpli Delpy, 1946). }
$$

\section{BIOLOGIE}

Selon Rousselot $(1948,1953)$ c'est une tique à trois hôtes. En A.O.F. elle est très fréquente dans tout le Sahel; chez les zébus elle se fixe, comme tous les Hyalomma, sur les parties inférieures : fanon, ars, ventre, mamelle, scrotum, périnée, marges de l'anus.

\section{HYGROPHILIE}

C'est par excellence une tique sahélienne; elle représente la plus grande partie des Hyalomma récoltés en cette région. Au nord de la région soudanienne, elle se trouve mêlée à $H$. rufipes et $H$. truncatum. En A.O.F. H. impeltatum est réparti entre les isohyètes des 100 et $1000 \mathrm{~mm}$. Les références plus au sud concernent des tiques prélevées sur des animaux d'abattoirs, ou amenées par des troupeaux venant du nord. Sa répartition recouvre, en A.O.F., une partie de celle des zébus.

\section{HOTES}

Les adultes se gorgent sur les grands mammifères du Sahel. On les a trouvés sur zébu, mouton, chèvre, dromadaire, cheval, gazelle, oryx, mouflon, caracal.

Les immatures se gorgent sur petits mammifères (voir $H$. dromedarii). Hoogstraal (1956) donne comme hôtes en Egypte les gerbilles, gerboises, psammomys, lièvres.

\section{DISTRIBUTION}

Proche Orient, Sudan Rep., Erythrée, Kenya, Tanganyika, (Hoogstraal, 1956) ; Iran (Delpy, 1949) ; Maroc et Tunisie (exemplaires dans les collections MHNP, CBpt, IPP : Harazin (Mogador), Gafsa, Douiret, Tatzouine, Kébili, Tozeur, El Hamma de Gabès); Tchad (Ati : IPP); Algérie ( $\mathrm{CNm}$ : Tébessa, Le Kreider, Akbès).

DISTRIBUTION DE Hyalomma impeltatum EN AFRIQUE OCCIDENTALE

\section{Dahomey :}

Malanville; Cotonou (abattoirs).

Côte D'lvoire :

Bouaké (Rousselot 1948 : abattoirs ?)

$$
\text { Haute Volta : }
$$

Sampelga (Dori).

\section{Rio de Oro:}

Le type de $H$. impeltatum provient d'une localité non précisée de ce territoire (Schulze et Schlottke, 1930 ; Kratz, 1940).

\section{Mauritanie :}

Atar (IFAN $=H$. rufipes glabrum de Villiers, 1955) ; Akjoujt : mouton ; Tidjikja : dromadaire ; Boutilimit : zébu, mouton, dromadaire, âne ; Boghé : dromadaire, zébu; Moundi (Boghé); Douera : zébu, dromadaire, âne (Moudjeria); Moudjeria : zébu, mouton, chèvre, dromadaire; mare de Touil : mouton; Aioun el Atrous : zébu, mouton, chèvre, dromadaire, cheval; el Beyed : mouton, zébu (Aioun) ; Tougoumbou : gazelle korine; Tamchaket: Togba (Tamchaket) ; Atar : hérisson ( $n$ : IFAN ; ou $H$. dromedarii); probablement Port Etienne (Nuttall. 1925 p.p.).

\section{Sénégal :}

Dagana : mouton (IPP) ; Podor : zébu, dromadaire (IPP) ; Dakar (CBpt).

\section{Soudan Français :}

Nioro : zébus, moutons; Bamako (Rousselot, 1951, 1953), zébus ; Banamba (IPP) ; Ségou (Rousselot, 1948, 1951, 1953);'Dougoufé(Ségou): Togo (IPP) ; Amba (Boré) ; Sansa (Gniminiama); Gogoro (Douentza); Tombouctou (Rousselot, 1948, 1951, 1953), (IPP) ; Bourem (IPP) ; Gao (Rousselot, 1948), (IPP) ; Agamor (MHNP : Bourem); In Rhar : oryx, In Ouri, oued Ifei (MHNP : Adrar des Ifoghas).

\section{Niger :}

Niamey ; dromadaire; Ayorou ; Tillabéri ; Ouellam : dromadaire; Filingué : dromadaire; Dakoro : zébu, mouton; Tessaoua ; Zinder; Myrria (Zinder); Nguigmi ; Babouloua (Nguigmi); Anou Araren, Marandet :-dromadaire, Bou Gouten : zébu, dromadaire, cheval ; Mt Baguezan; el Abiod (MHNP), kori Atkakit : caracal (Villiers, 1955) (Air); Agadès : chien $(n)$, In Gall : lièvre $(n)$ (ou $H$. dromedarii).

\section{Sahara :}

Tin Aberda : mouflon (MHNP); Timissao ; Silet : homme (Tamanrasset); oued Assassou oued Issandilen, oued Enfel, Timaksiouine, Aharar (IPP : Tassili des Ajjer $=H$. dromedarii p.p. de Colas-Belcour et Jacquemin, 1953) : Abalessa : hérisson $(n, l:$ MHNP ; ou $H$. dromedarii). 


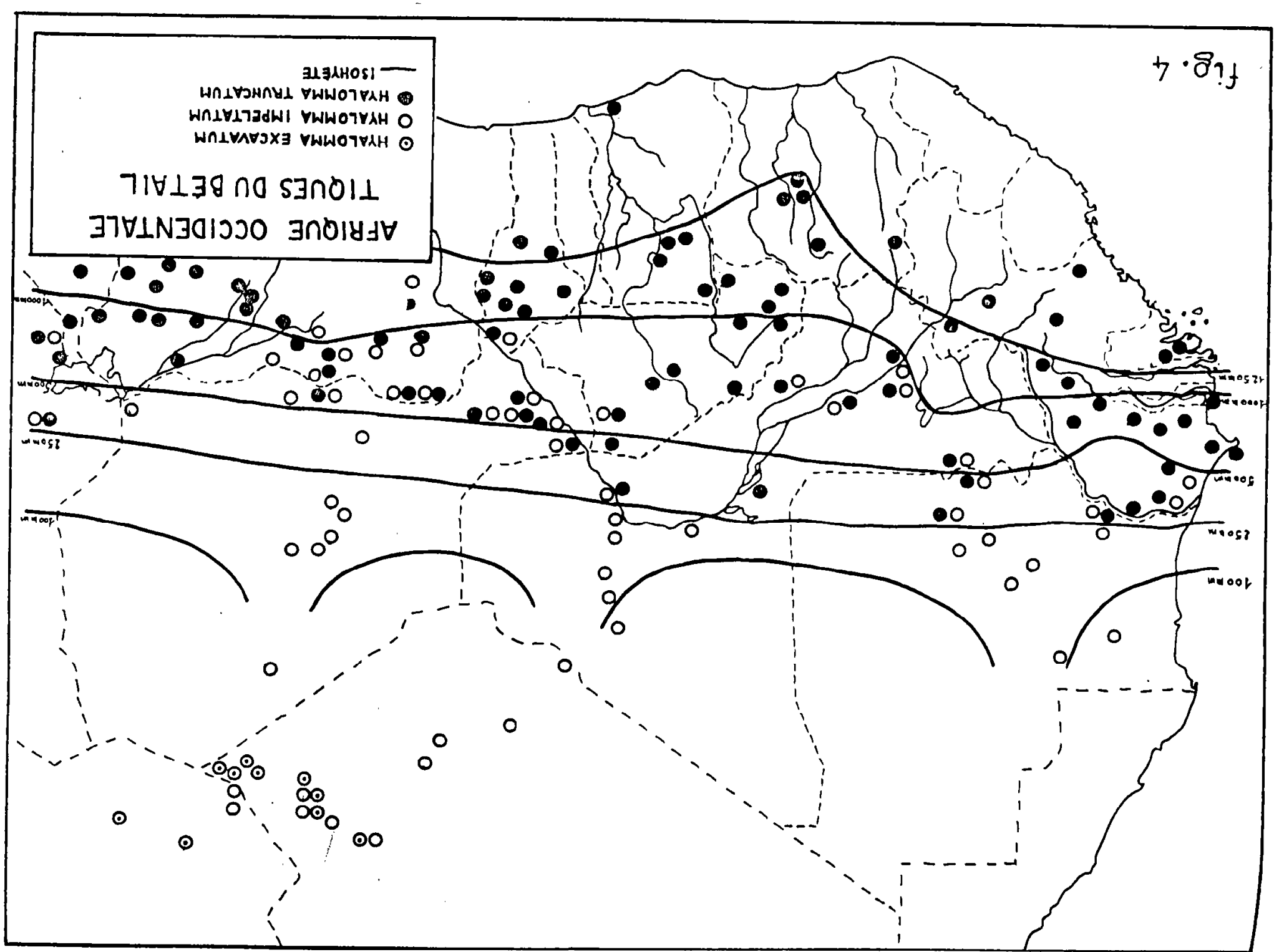




\section{Lybie :}

Bir Abaneur, Ghat (IPP : Tassili des Ajjer = Colas-Belcour et Jacquemin, $1953: H$. dromedarii p.p.).

140) Hyalomma impressum Koch, 1844.

Cette tique semble avoir à peu près la même biologie que $H$. rufipes. Morphologiquement voisines, ces deux especces sont souvent confondues dans la littérature jusqu’à ces dernières années. $H$. impressum est répandu dans les régions de savanes du Sénégal au Sudan Rep. (Hoogstraal, 1956). En A.O.F. il est présent entre les isohyètes de 500 et $1250 \mathrm{~mm}$, presque toujours mêlé à $H$. rufipes. Les adultes se fixent sur le bétail, les immatures probablement sur les oiseaux et les petits mammifères, comme $H$. rufipes. $H$. impressum semble légèrement plus hygrophile que ce dernier.

DISTRIBUTION DE Hyalomma impressum EN AFRIQUE OCCIDENTALE

Côte d'Ivoire :

Bouaké (abattoirs); Ndana (Katiola).

\section{Dahomey :}

Cotonou (abattoirs); Parakou; Malanville; Karimama.

Haute Volta :

Bobo-Dioulasso ; Samandéni ; Dargo (Kaya).

\section{Niger :}

Niamey : dromadaire; Tillabéri; Ayorou ; Ouellam : dromadaire; Filingué : dromadaire; Tessaoua.

\section{Sénégal :}

Sangalcam ; Keur Dembakélé : cheval (SCHMP : Thiès); Kédougou; Sédhiou.

\section{Soudan Français :}

Nioro ; Bamako : (Rousselot, 1951, 1953), zébus ; Gavignané (Nioro); Banamba (IPP): Ségou : (Rousselot, 1951, 1953), zébus ; Fambougou, Zangon Ouéré (Ségou); Dioro ; Bani : phacochère; Baguineda (Girard et Rousselot, 1945) ; Békuy (San) ;

\section{Tchad :}

Fort Archambault (MHNP).
150) Hyalomma rufipes Koch, 1844.

\section{BIOLOGIE}

C'est en général une tique à 2 hôtes, avec possibilité de cycles à 3 hôtes (Hoogstraal, 1956). (Il semble cependant que les références concernant cette dernière possibilité soient le fait d'auteurs qui ne distinguaient pas $H$. rufipes de truncatum : les expériences sont à reprendre). En A.O.F. nous avons trouvé sur oiseaux des formes immatures de $H$. rufipes (déterminées par l'élevage des nymphes) : larves et nymphes sur la tête, souvent près du conduit auditif. On pouvait remarquer :

a) des larves en nymphoses, fixées, contenant une nymphe;

b) des dépouilles larvaires implantées ;

c) des nymphes fixées, leur rostre contigu au rostre larvaire, la dépouille larvaire recouvrant comme un manchon le capitulum et les premières paires de pattes de la nymphe ;

d) des nymphes en évolution contenant des adultes.

On avait manifestement les 2 stades immatures sur le même hôte.

\section{HYGROPHILIE}

Cette espèce se trouve dans la zone de pluies annuelles qui sont de 300 à $1500 \mathrm{~mm}$. Les références les plus au sud concernent des exemplaires isolés, peut-être introduits par le passage de troupeaux du nord. En zone sahélienne et soudanienne cette tique est très abondante.

HOTE

Les adultes se gorgent sur zébus, bovins, mouton, chèvre, dromadaire, cheval, âne, hippotrague.

Les immatures évoluent sur les oiseaux, surtout des passereaux (Hoogstraal, 1956). En ce qui concerne l'A.O.F., il est certain que les mange-mil, déjà redoutables par eux-mêmes, jouent un grand rôle d'intermédiaires dans l'évolution de $H$. rufipes, ainsi que les francolins et d'autres espèces. Nous avons trouvé $H$. rufipes également sur petit calao, milan, tourterelle masquée, moineau.

\section{DISTRIBUTION}

Afrique au sud du Sahara, Madagascar, Proche-Orient, Russie (Hoogstraal, 1956). 
DISTRIBUTION DE Hyalomma rufipes EN AFRIQLE OCCIDENTALE

\section{Côte d'livoire :}

Mahandianarba (Odienné) ; Touba ; Bouaké (abattoirs); Afankaha (Katiola).

\section{Dahomey :}

Cotonou (abattoirs) ; Bouca (Nikki) ; Sondo (Kandi); Séfou : hippotrague; Malanville ; Karimama.

\section{Guinée Française :}

Metta (Dabola) : Siguiri : Fandia, Gbenkorokoro (Siguiri); Gada Bendoun (Lélouma); Dioulabougou (Kissidougou).

\section{Haute Volta :}

Silalla (Gaoua) ; Kpuéré : mouton (Batié) ; Moussodougou (Banfora) ; Bobo-Dioulasso; Banankélédaga (Bobo); Samandéni ; Kpakpara, Lokodja (Diébougou) ; Koumbara, Léri, Oué : cheval (SGHMP : Tougan); Ouagadougou : Yengo : zébu, cheval, Dargo, Zambamga (Kaya) ; Békouré, Garango : zébu, cheval (Tenkodcgo) ; Batibogou, Gountouré, Sampelga (Dori); Tin Akof : dromadaire.

\section{Mauritanie :}

Aleg; Mokhtar el Ajar (Aleg); Douera (Moudjeria) ; Tidjikja : dromadaire ; Boutilimit : dromadaire; mare de Touil : mouton; Aioun el Atrous : dromadaire; lac de Rkiz : mange-mil ( $n, l:$ IFAN ; ou impressum) ;

Atar (Villiers, $1955:$ H. rufipes glabrum) = impeltatum.

\section{Niger:}

Niamey : dromadaire; Tillabéri ; Ayorou ; Ouellam : dromadaire; Filingué : dromadaire; Dakoro : zébu, poulet ( $n$ renfermant un mâle); Tesszoua ; Zinder ; Myrrhia (Zirder) ; Nguigmi ; Babouloua (Nguigmi) ; Marardet : dromodaire (Agadès).

\section{Sénégal :}

Hann : milan ( $n$ ayant donné un mâle : Dakar) : Sangalcam ; Keur Dembakélé : cheval (SGHMP : Thiès) ; Louga : dromadaire; Guet : dromadaire (Sagatta); Dahra; Dagana (IPP); Bakel ; Kaolack; Keur Bouki (Kaolack); Kaffrine ; Mbayène (Koungheul) ; Tambakounda ; Kédougou ; Bantanani (Bala);

\section{Soudan Français :}

Nioro : zébus, moutons, cheval, moineau $(\mathrm{n}, l)$, tourterelle masquée $(n)$, petit calao $(n)$; Lorak Bane : cheval, francolin de Clapperton ( $n, l)$ (Nioro) ; Gavignané (Nioro) ; Bamako : (Rousselot, 1951, 1953), zébus ; Baguineda (Girard et Rousselot, 1945); Banamba (IPP) ; Kati (Giroud et coll., 1957) ; Ségou : (Rousselot, 1951, 1953), zébu, cheval; Dougoufé : zébru, cheval, Fambougou (Ségou); Togo (IPP); Niono : zébus, mange-mil ( $n$ ) ; Amba (Boré) ; Gniminiama ; Sansa (Gniminiama) ; Gogoro (Douentza) ; Ségou : traquet éthiopien (n) (Rousselot, 1953).

$$
\text { Togo : }
$$

Santé (Bassari).

\section{Sierra Leone :}

Kaballa.

\section{Nigéria :}

Unsworth (1952) cite beaucoup de points de récolte de l'espèce que d'autre part il ne distingue pas de $H$. impressum; il serait souhaitáble que son matériel soit examiné à nouveau.

\section{Tchad :}

Fort Lamy : mange-mil ( $n$ : IFAN) ; Ati : porc (IPP).

\section{6 $6^{\circ}$ Hyalomma truncatum Koch, 1844.}

$$
\text { (=H. transiens, Schulze, 1919). }
$$

\section{BIOLOGIE}

Tique probablement à trois hôtes. Les lieux de fixation des adultes sur les bovins et zébus sont les mêmes que pour les autres tiques longirostres : fanon, ars, mamelles, scrotum, périnée, pourtour de l'anus.

\section{HYGROPHILIE}

En A.O.F. l'aire de répartition est comprise entre les isohyètes de 500 et $1500 \mathrm{~mm}$, c'est-àdire dans les zones sahélienne et soudanienne. En région sahélienne $H$. truncatum est moins abondant que $H$. impeltatum. En revanche, en zone soudanienne c'est le plus fréquent des Hyalomuna, qui ne représentent plus d'ailleurs le genre dominant parmi les tiques du bétail comme c'est le cas au Sahel. En région guinéenne $H$. truncatum se raréfie notablement et les spécimens rencontrés ne le sont qu'en petit nombre à la fois. Il doit s'agir dans la plupart des cas 
d'introduction par des troupeaux du nord, ou de prélèvements dans des abattoirs. Des cinq espèces d'Hyalomma d'A.O.F. c'est $H$. truncatum qui s'accomode le mieux d'une certaine humidité. Il semble qu'il n'y ait qu'une génération par an, avec apparition massive des adultes dès le début de l'hivernage, ou même quelques temps avant. Au Sahel on en trouve toujours quelque peu en saison fraîche, alors qu'à ce moment l'espèce devient rare en zone soudanienne.

\section{HOTES}

Ceux des adultes sont les grands mammifères domestiques et sauvages. Par contre, comme dans la plupart des cas de parasitisme des tiques peu spécifiques, les immatures se gorgent sur les petits mammifères, et sur les oiseaux (mais non exclusivement sur ces derniers, comme cela semble le cas pour $H$. rufipes).

\section{DISTRIBUTION DE Hyalomma truncatum EN AFRI- QUE OCCIDENTALE}

\section{Cote d'Ivoire :}

Abidjan (abattoirs) : bovins, mouton ; Odienné ; Bouaké (abattoirs) ; Afankaha, Kationou ; Yékolo (Katiola) ; Korhogo.

\section{Dahomey :}

Cotonou (abattoirs) ; Parakou ; Nikki ; Bouca (Nikki) ; Gamia, Sikki, Zansarou, Séréké (Bembéréké) ; Pébéra (Béroubouay) : Djougou : Tanguiéta ; Gouandé (Tanguiéta); Angaradébou, Kofo, Sondo, Tioka, Toui (Kandi) ; Gogonou : Seghana ; Liboussou, Piami (Segbana) ; Séfou ; hippotrague; Soroko (Banikoara); Malanville; Karimama.

\section{Guinée Française :}

Kindia ; Gada Bendoun (Gaoual) ; Siguiri ; Limbana (Siguiri) ; Sareya ; Konkoronya, Thiankaye (Dabola).

\section{Haute Volta :}

Zinka : mouton, Silalla (Gaoua); Banfora (SGHMP) ; Mondon, Moussodougou, Tengréla. Tiéfora (Banfora) ; Bobo-Dioulasso ; zébu, lièvre ( $n$ : SGHMP) ; Banankélédaga (Bobo) ; Samandéni : Diébougou : hippotrague (SCHMP); Kpakpara (Diébougou); Houndé : hippotrague (SGHMP) ; Bissan, Foumani, Léri, Touroukoro : âne (SGHMP : Tougan) ; Ouagadougou ; Cissin, Sohotenga : guib (Ouagadougou); Dargo, Yengo : cheval Zambamga : mouton (Kaya); Békouré, Garengo (Tenkodogo) ; Pô : phacochère (IPP): Batobogou, Sampelga (Doru).

\section{Mauritanie :}

Boghé ; Moundi : mouton (Boghé) ; mare de Touil : mouton; Aioun el Atrous.

\section{Niger :}

Niamey : dromadaire; Tillabéri ; Ayorou ; Ouellam ; dromadaire; Filingué : dromadaire; Tessaoua ; Dakoro : Zébu, mouton, cheval, âne; Zinder.

\section{Sénégal :}

Dakar : hérisson ( $n$ : IFAN) ; Hann : effraie, Fann : hérisson, Tiaroye : hérisson (IFAN) (Dakar); Sangalcam (Rufisque); Thiès (SGHMP) ; Keur Dembakélé (SGHMP : Thiès) ; Louga : zébu, cheval; Linguère (IPP) Dahra; Dagana : mouton (IPP) ; Bakel ; Guet (Sagata); Niakhar : cheval ; Fatick ; Nakha Maye, Séssène (Fatick) ; Barkayel, Bouldiabé, Diokhoul, Kaville, Keur Bouki, Koutal, Mbadio, Ndobène, Ndoffane, Ouyal Sandé (Kaolack) ; Diamal, Hamdallaye, Ségré (Birkelane); Lama Fara, Lonkane (Kaffrine) ; Nganda ; Mbayène (Koungheul) ; - Nioro du Rip ; Tambakounda (IPP) ; NiokoloKoba: phacochère (Morel, 1956) ; Kédougou ; Doubirou, Sambatara, Saré Sori, Simbi (Vélingara).

\section{Soudan Français :}

Nioro : zébu, mouton, cheval, francolin de Clapperton ( $n$ ); Lorak Bane : phacochere (Nioro) ; Gavignané (Nioro) ; Bamako : (Rousselot, 1951, 1953), zébus ; Kati (Giroud et coll., 1957) ; Sotuba ; Banamba (Girard et Rousselot, 1945); Ségou : (Rousselot, 1951, 1953), zébu, cheval; Dougoufé, Fambougou, Sanando, Sotabougou, Zangon Ouéré (Ségou) ; Bani ; Dioro ; San; Békuy, Gioutiou (San); Togo (IPP); Niono (Giroud et coll., 1957); Amba (Boré); Gniminiama ; Sansa (Gniminiama) ; Gogoro (Douentza) ; Niafunké : phacochère (MHNP); Gao : porc (IPP).

\section{Togo :}

Nataré, Tamantougou (Naki-est) ; Dapango ; Cinkassé, Nadingou (Dapango).

\section{Gambie :}

Alijamadu : chien, Bakau, Albreda (Simpson, $1911:$ H. aegyptium). 


\section{Guinée Portugaise :}

Cacheu, Babanda, Caroncâ (Tendeiro, 1948, 1951, 1952, 1955).

\section{Gold Coast-Ghana :}

Zantana : hippotrague, Penyabi : phacochère (Simpson, 1914); Larabanga : hippotrague, Daboya : phacochère. (Simpson, 1918); Accra (Macfie, 1915); Kumasi, Tamale, Bawku (Moody, 1922).

Toutes ces références sont citées sous le nom de $H$. aegyptium. Comme certaines de ces localités sont situées au nord du territoire, il est fort probable que les auteurs avaient affaire non seulement à $H$. truncatum, mais encore à $H$. rufipes ou $H$. impressum.

\section{Nigéria :}

Nombreuses localités citées par Unsworth, 1952.

\section{Tchad :}

El Hamis (MHNP).

\section{0) ESPECES DU GENRE Ixodes.}

Les espèces de ce genre sont, en Afrique Occidentale, des parasites d'animaux sauvages de forêt ou de savanes très humides. Leur spécificité par rapport aux hôtes est très marquée. Le parasitisme des animaux domestiques est exceptionnel. Contrairement à ce qui se passe en Europe, ce genre est sans importance par rapport au bétail en A.O.F. En Afrique orientale et australe, $I$. cavipalpus et $I$. pilosus ont par ailleurs une importance certaine.

I. rasus (sensu lato) a été signalé sur chien à Obuasi (Nuttall et Warburton, 1911), sur bouf à Dayes (Togo) (Rousselot, 1951, 1953). Une nymphe d'I. ugandanus, parasite spécifique do l'aulacode (rongeur) est également citée sur mouton d'Ilesha (Nigeria : Nuttall et Warburton, 1911). Toutes ces déterminations doivent être remises en question, car il se trouve que ces espèces font partie d'un groupe rasus-ugandanus comprenant des formes bien caractérisées mais très voisines, dont nous aurons une comnaissance exacte seulement lors de la publication prochaine d'Arthur.

189) Rhipicephalus capensis longus Neumann, 1907.
Cette tique, abondante en savane humide d'A.E.F., est absente d'A.O.F. Les trouvailles les plus proches concernent une localité du Cameroun Britannique (Unsworth, 1952 : Sabga) Les types de $R h$. falcatus Neumann, 1908 sont des synonymes de longus. Leur origine est double : Rhodésie et Libéria. Or Neumann avait reçu ces derniers exemplaires dans un lot prélevé sur animaux sauvages du Jardin Zoologique de Leyde, et qui, en partie seulement, devaient provenir du Libéria. C'est plus qu'il n'en faut, jusqu'à plus ample information, pour faire douter de l'exactitude de cette origine pour $R h$. falcatus (Neumann, in tab.). (Pour Nuttall et son école $R h$. falcatus désignait $R h$. simus senegalensis Koch).

190) Rhipicephalus compositus Neumann, 1897 ,

Cette tique, du groupe capensis, est spécifique du buffle en région montagneuse et hauts plateaux. Unsworth (1952) la cite de localités du plateau central de Nigéria, sur bovins, sous le nom de $R$ h. ayrei Lewis, 1933. Donitz (1905) la signale de Bismarckburg, mais il ne précise pas s'il s'agit du Togo ou du Tanganyika, où existc une ville homonyme.

Nous n'avons pas trouvé l'espèce durant la prospection en A.O.F.

\section{$20^{\circ}$ ) Rhipicephalus evertsi evertsi ( $\mathrm{Neu}^{-}$ mann, 1897).}

\section{BIOLOGIE}

Son évolution se poursuit sur deux hôtes seulement (fait exceptionnel chez les rhipicéphales). Son lieu de fixation préférentiel semble être les marges de l'anus des équidés pour les adultes, pour les immatures le comet auriculaire.

\section{HYGROPHILIE}

Les renseignements à ce sujet en A.O.F. sont rares, mais en les comparant à ceux d'Unsworth pour la Nigéria les limites de répartition scmblent être les isohyètes de 500 et $1000 \mathrm{~mm}$.

C'est une tique en général assez abondante sur les animaux qu'elle parasite. Il doit n'y avoir qu'une génération par an, dont les adultes apparaissent en saison froide (fin octobre, décembre et février pour les échantillons reçus).

HOTES

L'hôte d'élection de l'adulte est le cheval, 


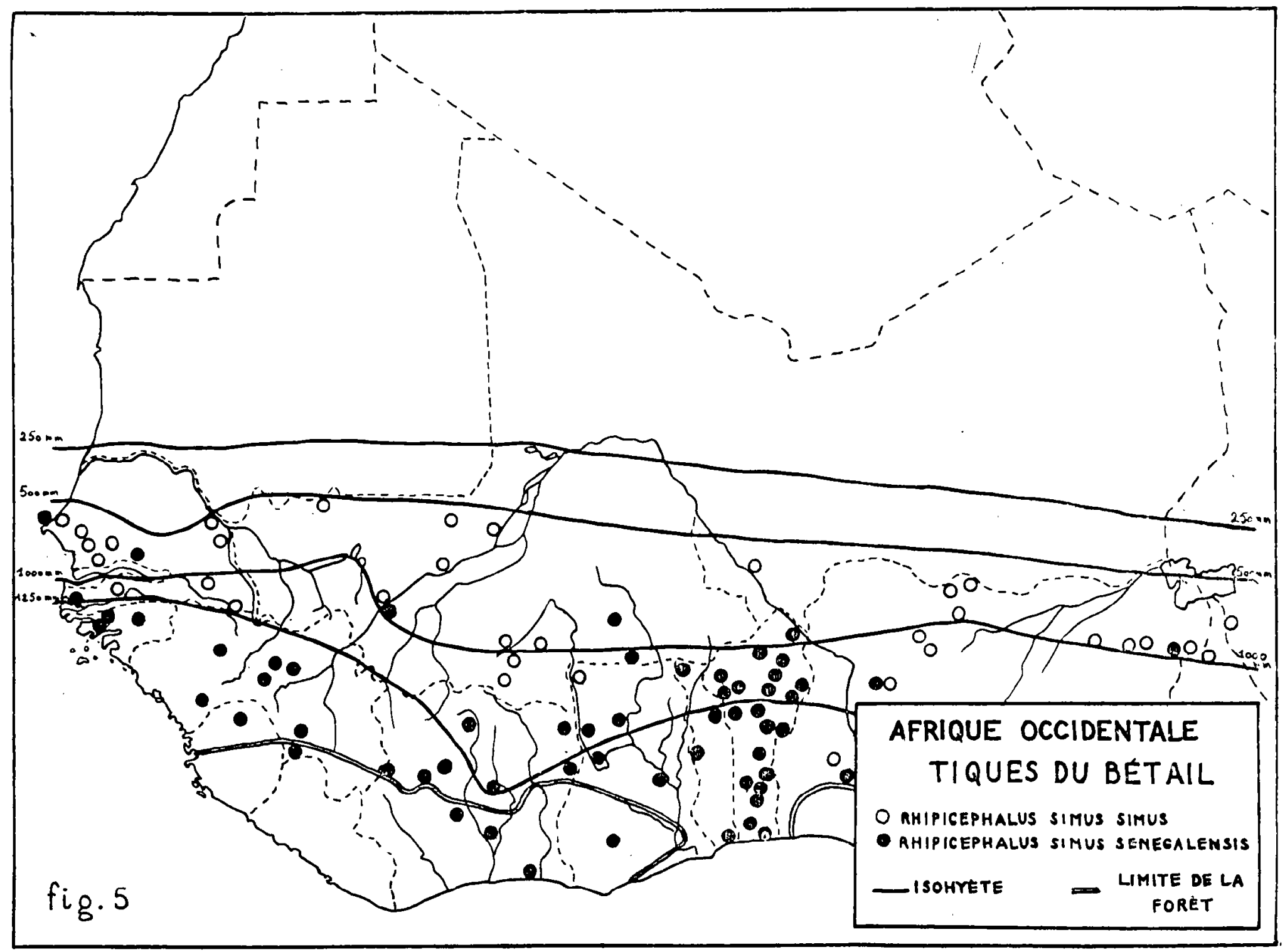


puis l'âne. Bovins, moutons, chèvres ne sont parasités que d'une façon secondaire. En Afrique orientale et australe les auteurs citent comme hôtes de nombreux herbivores sauvages. Le cas ne s'est pas produit en A.O.F. Nous supposons d'ailleurs que $R h$. evertsi y est d'introduction artificielle, amené avec les chevaux d'Afrique orientale, peut-être au cours des migrations peules vers l'ouest.

Les immatures se fixent sur les mêmes hôtes que les adultes. Cette tique est donc dans de bonnes conditions pour jouer un rôle dans la transmission de piroplasmoses et theilerioses, surtout chez le cheval. Cette tique est en effet un important vecteur : Piroplasma bigeminum, $P$. caballi, Nuttallia equi, Theileria parva, Th. mutans, Th. ovis.

\section{DISTRIBUTION}

Dans toute l'Afrique au sud du Sahara, mais avec une abondance variable (surtout Afrique orientale et australe).

DISTRIBUTION DE Rhipicephalus evertsi EN AFRIQUE OCCIDENTALE

Sénégal :
Louga : cheval.

\section{Soudan Français :}

Bamako : zébu, chèvre, cheval, âne, chien, Ségou (Rousselot, 1951, 1953) ; Baguineda (Girard et Rousselot, 1945).

\section{Haute Volta :}

Oué : cheval (SGHMP : Tougan); Garango : zébu, cheval, mouton (Tenkodogo); Bagré : mouton (Tenkodogo).

\section{Togo :}

Lomé : cheval, chèure (Neumann, in tab.); Togo (Ziemann, 1905).

\section{Niger :}

Maradi : cheval (IPP).

\section{Nigéria :}

Nombreuses références pour les provinces du nord (Unsworth, 1952).

\section{0) Rhipicephalus (Pterygodes) fulvus (Neumann, 1913).}

Cette tique, connue uniquemert jusqu'à ce jour du Sud tunisien, parasite à l'état larvaire et nymphal les petics rongeurs désertiques (goundis) et à l'état adulte divers grands mammifères : dromadaire, chèvres, moutons (Colas-Belcour, 1932 ; Colas-Belcour et Rageau, 1951) et probablement la gazelle dorcas et le mouflon. Nous en avons examiné des exemplaires provenant de divers massifs montagneux sahariens qui semblent d'ailleurs constituer leur habitat normal :

Mt Baguezan (Air) : goundi ( $n$ ), un mâle sur le sol (IFAN) oued Koudou (Tibesti : $1800 \mathrm{~m})$ : goundis $(\mathrm{n})$; Tibesti $(3000 \mathrm{~m})$ : une femelle sur le sol.

Il serait donc intéressant de rechercher l*s adultes sur ruminants domestiques et sauvages dans les régions citées (Niger et Tchad).

\section{0) Rhipicephalus pravus (Dônitz, 1910).}

C'est une espèce proprement d'Afrique orientale. Les localités citées par Unsworth (1951) en Nigéria (Bakanke, Jakiri) sont inattendues. Simpson (1912) avait rapporté $R h$. neavei Warburton, 1912 d'Oshogbo, et $R h$. appendiculatus Neumann 1897 de Zungeru. Rh. neavei est synonyme de $R h$. pravus (Hoogstraal, 1956 et Walker, 1956) et dans le cas de Rh. appendiculatus il y a peut-être eu confusion avec d'autres espèces (un $R h$. sanguineus très ponctué). $R h$. pravus n'a pas encore été trouvé en A.O.F.

230) Rhipicephalus sanguineus (Latreille, 1806).

BIOLOGIE

C'est une tique à trois hôtes, dont les adultes se gorgent sur de nombreuses espèces de mammifères, et les immatures sur rongeurs et oiseaux.

Cette espèce qui, sur les animaux sauvages, n'est pas plus abondante que d'autres, voit son développement favorisé dans des conditions particulières telle que l'adaptation à des animaux domestiques comme le chien. La tique trouve alors dans les abris artificiels divers (sombres et humides) : niches, fentes de murs, etc... les conditions d'une véritable pullulation. La tique est devenue semi-domestique. On a signalé à plusieurs reprises l'envahissement de l'habitation humaine elle-même.

Les lieux de fixation sur mammifères sont divers, mais ce sont plus habituellement les oreilles, surtout à l'intérieur du pavillon. Ceci est net chez le chien, les petits ruminants, et les bovins chez qui nous en avons trouvé en grand nombre au Dahomey, en compagnie de $R h$. simus. 
Les petits ruminants sont infestés entre les doigts. Les femelles restent fixées en moyenne une semaine, parfois deux ou trois.

Dans les conditions semi-domestiques, $R h$. sanguineus semble avoir plusieurs générations au cours de l'année car on le trouve tout le temps. A l'état sauvage, on assiste comme dans le cas des autres espèces à l'explosion numérique d'hivernage en région soudanienne. Le fait est moins net en région guinéenne. Dans n̈importe quelle région, ou peut trouver à tout moment de l'année $R h$. sanguineus, en plus ou moins grande abondance, il est vrai, mais il n'est jamais absent.

\section{HYGROPHILIE}

$R h$. sanguineus se rencontre aussi bien au nord du Sahel qu'en région guinéenne. Dans le premier cas il faut tenir compte d'un biotope artificiel péridomestique, ce qui atténue les conditions réelles de sécheresse du pays considérć. Il semble donc que dans les conditions naturelles l'espèce se maintienne entre l'isohyète de $500 \mathrm{~mm}$ et la forêt, où, sur animaux sauvages, il est remplacé par $R h$. ziemanni. Au début de l'enquête en A.O.F., en relation avec la répartition publiée en Nigéria, il semblait qu'en région guinéenne l'espèce devenait plus rare; de toute façon la plupart des échantillons reçus provenaient de chien et, en région sahélienne, de moutons. Les bovins semblaient exceptionnellement parasités (fait que les auteurs signalent pour beaucoup de régions hors d'A.O.F.). Or, au cours d'une mission au Dahomey nous avons pu constater que les $R h$. sanguineus étaient très nombreux mais localisés à l'intérieur des oreilles presque uniquement, au point qu'on pouvait les y trouver à coup sûr. Les collecteurs, l'attention attirée par les tiques du fanon, du périnée, etc... négligent souvent les oreilles. Peut-être est-ce à une raison semblable qu'il faut attribuer l'apparente rareté de l'espèce en Nigéria (Unsworth, 1952).

On constate donc que $R h$. sanguineus se maintient au long d'une échelle assez étendue de pluviosités. Il semble cependant qu'il y ait eu adaptation de souches qui se traduit par une variation morphologique. L'espèce est très variable dans sa taille et ses ponctuations. La forme typique périméditerranéenne se retrouve au Sahel et en rćgion soudanienne nord; les ponctuations du scutum sont de deux sortes : ponctuations pilifères grosses, en ligne, et ponctuations interstitielles fines. En région sahélienne sud, les deux sortes de ponctuations s'égalisent et le scutum tend à devenir uniformément ponctué (en même temps que la taille diminue). Ainsi la différenciation de ces deux formes traduirait l'adaptation à des milieux hygrométriquement différents.En A.O.F. la ligne probable de passage d'une forme à l'autre est constituée par l'isohyète de $1000 \mathrm{~mm}$.

Telle sont les conclusions que l'on peut tirer des faits pour l'A.O.F. Hoogstraal (1956) signale également qu'au Sudan la forme très ponctuée ne se trouve qu'au sud.

On a donc affaire dans le cas de $R h$. sanguineus à une différenciation analogue à celle des sousespèces de $R h$. simus, mais du point de vue systématique la question n'est pas résolue.

Hotes (en Afrique occidentale).

Adultes : zébus, bovins, mouton, chèvre, chien, chat, cheval, âne, porc, homme, singe rouge, chacal (Canis anthus et $C$. adustus), renard, hyène, serval, chat sauvage, civette, zorille, mangouste, panthère, lion, phacochère, gazelle, guib, ourebie, sylvicapre, bubale, hippotrague, hérisson (Atelerix abliventris et Paraechinus aethiopicus), lièvre, rat de Gambie, autruche, grande outarde, vautour ( $P$ seudogyps africanus), effraie, milan.

Immatures : ils se gorgent sur petits mammifères, mais également sur les mêmes hôtes que les adultes : chien, chacal, galago, rat roussard.

\section{DISTRIBUTION}

Cette espèce, originairement africaine et périméditerranéenne (où elle parasite les animaux sauvages) s'est répandue à la suite du chien, par le monde entier (cf. Leeson, 1953).

DISTRIBUTION DE Rhipicephalus sanguineus EN AFRIQUE OCCIDENTALE

\section{Côte d'lvoire :}

Abjijan : chien, mouton; Toumodi : chien (IPP) ; Bouaké : mouton et chèvre (CBpt), lièvre (Villiers, 1955) ; Korhogo : bovins, chien..Bouna : homme (SGHMP).

\section{Dahomey :}

Cotonou : bovins, porc ; Agblangandan (Cotonou) ; Porto Novo ; Atchoukpa, Pobé, Tohoué : chien (SGHMP) (Porto Novo) ; Dan (Bohicon) ; Atchéribé : céphalophe roux (IPP) ; Paouignan : bovin, chien, (Dassa) ; Dassa Zoumé ; Logozohé (Savalou); Savalou; Djalloukou (Tchetti) ; Agouagon : chien (IPP), liève (IPP); Gobada (Savalou); Savé ; Carnotville : chien ; Parakou : bovins, chat, liève; ferme Okpara; Bétérou, Yérémarou : chien (Parakou); Bori : lièvre 
(Ndali) ; Nikki : bovins, mouton; Biro, Koni : mouton, Kalale, Bessassi (Nikki) ; Gamia, Nagayérou, Passara (Bembéréké) Djougou ; Kouandé ; Natitingou ; Koussokoingou : lièvre (Villiers, 1955), Toukountouna : rat de Gambie (Natitingou); Gogonou : mouton, Kofo, Sondo, Toui, Angaradébou (Kandi) ; Séfou : bubale; Kouté : chien (Segbana); Malanville : chien; Goroubéri : chien (Guéné).

\section{Guinée Française :}

Conakry (Blanc et Coll., 1937, 1938) ; Pastoria ; Friguiagbé (MHNP) ; Telimélé : chien; Kankan : bovin, chien; Yogbotou : chien (Nzérékoré).

\section{Haute Volta :}

Gaoua : chien; Dankana : chien, mouton, Kpuéré : chien, Djigoué : chien (SGHMP : Gaoua); Banfora (SGHMP); Mangodaia chien, Sitiéna : chien, Tengréla : chien, Tiéfora : chien, chèure (Banfora); Bobo-Dioulasso : chien, hérisson (SGHMP), singe rouge (SGHMP), lièvre (SGHMP), gazelle (SGHMP), sylvicapre (SGHMP), panthère (SGHMP) ; Santidougou : lion (SCHMP), Banankélédaga, Koriba : homme (SGHMP), Karankasso : homme (SGHMP), Sinorosso : lièvre (SGHMP), (Bobo) ; Toussiana : hérisson (SGHMP); Samandéni ; Dougoumato : sylvicapre (SGHMP: Houndé); Kotédougou : gde outarde (SGHMP); Ouakuy : hippotrague (IPP) ; Nouna : âne (SGHMP) ; Sono : sylvicapre (SGHMP : Dédougou); $\mathrm{Di}^{\mathrm{i}}$ : Âne, Koumbara : chien, lièvre, Lanfiéra : chien, Niassan : homme, Oué : âne, chien, Touroukoro : âne, chien (SGHMP : Tougan); Ouagadougou : chien; Garango : mouton (Tenkodogo); Pô : phacochère (IPP); Fada-Ngourma : chien; Dori : chien.

\section{Mauritanie :}

Fort Gouraud : renard (Villiers, 1955) ; Atar : hérisson (Villiers, 1955); Douera : âne (Moudjeria); Bateleyed : gde outarde, Sabou Alla (Brakna); Boghé ; Moundi : mouton (Boghé); Aioun el Atrous; Oualata (MHNP).

\section{Niger :}

Niamey : chien; Tillabéri : zébu, mouton; Ayorou ; Zinder : chien; Dunkass : hérisson (IPP : Zinder); Agadès : renard (Villiers, 1955), chien.

Sénégal :

Dakar : chien, zorille (Villiers, 1955), rat noir ( $n$ : IPP); Hann : renard (Villiers, 1955), zorille (Villiers, 1955), effraie, milan, Yoff : chacal, Tiaroye : hérisson (IFAN) (Dakar); Sangacalm : buvins, chien, chat (Rufisque); Mbour : chat (SGHMP) ; Ngazobil : homme, Sandiara : serval, Dangane : chien, Ndiémané : lièvre (Mbour); Joal : homme ; Sébikotane : serval (Villiers, 1955); Thiès : hérisson (Villiers, 1955) ; chien (SGHMP); Louga : chat sawvage (Villiers, 1955), chien; Saint Louis ; chien (Neumann, 1897); Dahra : moutons; Dagana : chien, mouton (IPP) ; Podor : chien (IPP) ; Bakel : galago (Villiers, 1955) ; Souli (CBpt : Bakel); Dioin (Bambey); Birkelane : chien; Tambakouda : chien (IPP) ; NiokoloKoba : civette, ichneumon, chacal, guib, redunca, ourébie, lièvre (Morel, 1956) ; Kèdougou : chien; Boudhié (Sédhiou); Bignona : lièvre (Villiers 1955); Ziguinchor : chicn, mouton (IPP).

\section{Soudan Français :}

Nioro : zébus, moutons, chèvre, cheval, chat, chacal, vautour, rat roussard ( $n$ ) ; Lorak Bane : mouton, cheval, phacochère, civette, serval, Gavignané (Nioro); Bamako : (Rousselot, 1951, 1953), zébus; Sotuba; Sikasso : panthère; Ségou : chien, cheval, mouton, chèvre, gazelle, chacal, renard, hérisson, liévre (Rousselot, 1951. 1953), Ségou : zébu, mouton ; Togo (IPP) ; Niono: mouton (Giroud et coll., 1957), zébu, autruche; Sokolo : zébu, hyène; Ténenkou : âne, mouton; Djenné : lièure (IPP); Niafunké : phacochère (MHNP) ; Tombouctou : chien (IPP); Cogoro : chacal, hyène (Douentza); Amba : chacal (Boré) ; Sansa (Gniminiama); Kaore : chien, Kandé : chien (SGHMP : Bai).

\section{Togo :}

Lomé : chien; Sawaga (Naki-est) ; Komah : cheval (Sokodé); Togo (Neumann, 1897, 1901)

Gambie :

Alijamadu : chien (Simpson, 1911).

\section{Guinée Portugaise :}

Tendeiro (1948, 1951, 1952) rapporte l'espèce comme présente presque partout, sur bovins, mouton, chien, cheval, céphalophe de Maxwell, chien, chat, serval, panthère, ichneumon, civette, lièrere.

\section{Sierra Leone :}

Kaballa, Kanakwie, Laminaia, Yiraia, Panguma, Songo-Town : chiens dans toutes ces localités (Simpson, 1913); Kaballa : bovin.

\section{Gold Coast-Ghana :}

Accra (Macfie, 1915); Obuasi : chien (Neumann, in tab.); Kete Krachi : chien (CNm); 
Larabanga : hippotrague, Daboya : hippotrague, Curipe : sylvicapre, Yapei : chat sauvage (Simpson, 1918).

\section{Nigéria :}

Simpson (1911, 1912) et Unsworth (1952) citent de nombreuses localités pour cette espèce dont les hôtes sont divers animaux domestiaues: chien, mouton, chèvre, bovins.

\section{Tchad :}

Dïintilo (embouchure du Chari : MNHP); Ati : chien (IPP).

\section{Sahara :}

Tindouf : hérisson (IPP).

\section{$24^{\circ}$ ) Rhipicephalus simus (Koch, 1844).}

\section{BIOLOGIE}

Le cycle de cette espèce comprend trois hôtes. Les immatures se gorgent sur des rongeurs. C'est dans leurs terriers que larves et nymphes trouvent les conditions nécessaires à leurs mues. On y trouve très souvent des adultes nouvellement éclos. Puis les adultes s'échappent et gagnent des herbes (surtout graminées), où ils grimpent et attendent des mammifères de grande taille (ongulés, carnivores, etc...). D'après les observations en A.O.F., les adultes se fixent presque exclusivement dans les oreilles, où ils sont parfois très abondants (dans certains cas ils étaient plus d'une centaine et en tapissaient tout l'intérieur). Il semble qu'ils cherchent à se planter entre des poils dressés, et plus gros que le pelage normal (crins). C'est ainsi qu' on a pu les trouver souvent entre les poils de la crinière ou du toupillon de la queue. Chez les chiens on les trouve souvent sur les oreilles ou entre les yeux.

\section{HYGROPHILIE}

$R h$. simus est rare en région sahélienne-sud. Lorak Bane (Nioro) est un fond de vallée avec cours d'eau temporaire mais où demeure toujours une certaine humidité grâce à une importante galerie d'arbres (forêt classée). Autour de Nioro même le paysage est typiquement sahélien : il n'y a pas été trouvé de $R h$. simus. L'espèce augmente en densité en région soudanienne, et devient très abondante en région guinéenne dans la savane. En forêt, elle doit se limiter dans les clairières autour des villages. On rencontre approximativement $R h$. simus au sud de l'isohyète de $500 \mathrm{~mm}$. Les exigences hygrométriques sont donc assez larges. Mais l'espèce se scinde alors en deux sous-espèces aux exigences différentes de ce ploint de vue.

a) Rh. simus simus Koch, 1844 habite le sahel et la région soudanienne nord, jusqu' à l'isohyète de $1000 \mathrm{~mm}$.

b) Rh. simus senegalensis Koch, 1844, présent dans les zones qui reçoivent plus de $1000 \mathrm{~mm}$ de pluies annuelles. La distinction des sousespèces, basées sur la morphologie, est ici très bien corroborée par la biologie.

Cette répartition peut souffrir des exceptions, lorsqu' on a un îlot plus humide au sein d'une zone soudanienne. Ainsi, au Sénégal, Sangalkam, qui est une relique de végétation guinéenne avec cours d'eau, rômiers, palmiers, etc... ne reçoil pas plus de pluies que le reste de la région, mais l'importante couverture végétale y entretient un micro-climat extrêmement humide (gîte à Glossinga palpalis) où on trouve Rh. simus senegalensis alors qu'à Thiès c'est $R h$. simus simus qu' on rencontre.

$R h$. simus est une tique de région à végétation herbacée dense; l'espèce est particulièrement abondante dans la savane boisée du moyen et nord Dahomey au nord de la Côte d'Ivoire (savane ouverte) et sud de la Haute-Volta (savane boisée).

Unsworth (1952) écrit que les deux sousespèces ont à peu près la même répartition. Or il cite fort peu do prélèvements du sud de la Nigéria (cf. résultats des prospections au Dahomey).

\section{HOTES}

Adultes de $R h$. simus simus: zébus, bovins, cheval, âne, guib, redunca, ourcbic, phacochère, potamochère, porc épic, chacal à flancs noirs, civette, chien, hyène, lion.

Adultes de $R h$. simus senegalensis; bovins, mouton, cheval, porc, chien, buffle, hippotrague, bubale, lièvre, taupe, porc-épic, civette, panthère.

Nous n'avons pas trouvé d'immatures en A.O.F.

\section{DISTRIBUTION}

Afrique au sud du Sahara, Yemen (Hoogstraal, 1956).

DISTRIBUTION DE Rhipicephalus simus EN AFRIQUE oCCIDENTALE

\section{Côte d'Ivoire :}

Rhipicephalus simus senegalensis.

Abidjan : chien (IPP); Touna (MHNP : 
Haute Sassandra); Bouaflé (Villiers, 1955); Touba : Séguéla ; Mankono : Toumodi : chien (IPP) ; Tonhoulé, Minankro (Bouaké) ; Bouaké ; Bondoukou (IPP) ; Korhogo : bovins, chien; Cansé (SGHMP : Bouna).

\section{Dahorney :}

Rhipicephalus simus senegalensis.

Cotonou : bovins, porc; Agblangandan (Cotonou) ; Porto Novo ; Sémé (Porto Novo) ; Hountagbé (Athiémé); Djidja : chien (Ábomey) ; Dan (Bohicon); Atchéribé : céphalophe roux (IPP) ; Paouignan : bovins, chien (Dassa); Dassa Zoumé ; Logozohé (Savalou) ; Savalou ; Gnoukpuignon, Monkpa (Savalou) ; Djalloukou (Tchetti); Agouagon : bovins, cheval (IPP) ; Savé ; Kilibo ; Toui (Kilibo) ; Parakou ; ferme Okpara ; Yérémarou, Guéma (Parakou); Bétérou ; Bori : buffle (Ndali) ; Nikki ; Biro, Koni. Kalalé, Bessassi (Nikki) ; Gamia, Sékéré (Bembéréké) ; Ina ; Frékou (Béroubouay) ; Djougou ; Kouandé ; Natitingou ; bovins, cheval; Tanguiéta ; Gouandé (Tanguiéta) ; Gogonou : mouton (Kandi) ; Séfou : bubale; Banikoara : mouton; Toussou (Banikoara); Malanville.

\section{Guinée Française :}

Rhipicephalus simus senegalensis.

Kindia : buffle (IPP) ; Soundougou (Diari); Sareya ; Toumanea (IPP); Kodin (Dabola); Bélissa, Ouendékéra, Dioulabougou (Kissidougou); Soumdou (Gueckédou); Nzérékoré : porc; Gogota (Nzérékoré).

\section{Haute Volta :}

Rhipicephalus simus simus.

Bobo-Dioulasso : panthere (SGHMP) ; Santidougou : lion (SGHMP), Banankélédaga (Bobo); Samandéni ; Tiéfora : chien (Banfora).

Rhipicephalus simus senegalensis.

Ouagadougou : chien; Pô : phacochère (IPP).

Sénégal :

Rhipicephalus simus simus.

Sangalkam (Rufisque); Thiès : chien; Niakhar : cheval, Diassane : chacal (Thiès); Hamdallaye (Birkelane); Nganda ; NiokoloKoba : guib, redunca, ourebie, potamochère, chacal, civette, (Morel, 1956), porc-épic ; Kédougou : zébu, civette (IPP) ; Nayes : civette (IFAN) ; Souli (CBpt : Bakel); Sédhiou (SGHMP).

Rhipicephalus simus senegalensis.
Ile des Madeleines (Dakar : Villiers, 1955); Sangalkam ; Keur Bouki (Kaolack); Mbayène (Koungheul); Ziguinchor : chien; Sénégal (Koch, 1844).

\section{Soudan Français :}

Rhipicephalus simus simus

Lorak Bane : porc-épic, civette (Nioro); Bamako : (Rousselot, 1951, 1953), zébus; Sotuba ; Ségou (Rousselot, 1951, 1953) ; Sokolo: hyène; Ténenkou : âne; Sansa (Gniminiama) ; San (MHNP).

Rhipicephalus simus senegalensis.

Bamako, Ségou (Rousselot, 1951, 1953) ; Sotuba : quelques exemplaires; il est probable que la sous-espèce a été introduite avec du bétail de Guinée ou Côte d'Ivoire (cf. également à Sotuba la présence de Boophilus annulatus). Nous ne savons pas ce que représente exactement le matériel de Rousselot ; il est certain cependant qu'une partie de ses échantillons est constituée. par une autre espèce : Rhipicephalus simpsoni Nuttall, 1910, pris sur aulacode, dont c'est l'hôte typique (exemplaires examinés à l'IPP, dans la collection de tiques du Soudan, déposée par Rousselot lui-même).

\section{Togo :}

Rhipicephalus simus senegalensis.

Lomé : chèvre (Neumann, in tab.) ; Misahöhe (Neumann, in tab.); Bismarckburg : cheval (Zumpt, 1943 : type de $R h$. simus longoides); Bafilo (Sokodé) ; Léon : crocidure (Niamtougou); Nataré, Sawaga (Naki-est) ; Kabou, Santé (Bassari) ; Togo (Neumann, 1901 ; Ziemann, 1905).

\section{Guinée Portugaise :}

Les exemplaires de $R h$. simus mentionnés par Tendeiro (1948, 1951, 1952) appartiennent très probablement à la sous-espèce senegalensis. Bissau, Bissora et Canchungo : bouf, mouton, chève, cheval, porc, hippotrague, oryctérope, porc-épic, chien, civette, panthère.

\section{Sierra Leone :}

Hangha : chien (Simpson, $1913: R h$. simus : il doit s'agir en fait de la forme senegalensis); Kaballa : bovins ; Sierra Léone (Zumpt, 1943) (Ces deux dernières références concernent $R h$. $s$. senegalensis).

\section{Gold Coast-Ghana :}

Obuasi (Neumann, in tab.) : 'Zantana : phacochère, Penyabi : phacochère, Bandewa : ourébie, 
Mankuma : redunca (Simpson, 1914 : Rh. simus; il est possible quiune partie des récoltes soit du senegalensis); Daboya : phacochère (Simpson, $1918: R h$. falcatus $=$ s. senegalensis) ; Kete Krachi (Zumpt, 1943).

\section{Nigéria :}

Rhipicephulus simus simus.

Ilorin, Bida, Kontagora, Abuja, Fontua, Daudawa, Kano, Katsina, Tambu, Potiskum, Auno, Maidugari, Bama, Biu, Uba, Shellen, Numan, Toango (Unsworth, 1952).

Rhipicephalus simus senegalensis.

Bokani : chien, Zungeru, Tegina, Kontagora : cheval, Osubu, Baro (Simpson, 1911 : Rh. simus; Hoogstraal, 1956); Mina (Simpson, 1911 : Rh. falcatus) ; Ajasse, Bama, Biu, Mubi, Kiri (Unsworth, 1952).

\section{Tchad :}

Fort Archambault, Dai (MHNP : Rh. simus simus).

\section{Oubangui Chari :}

Fort Sibut : bovins, mouton, chèvre, porc (IPP : Rh. simus simus).

25) Rhipicephalus tricuspis Dönitz, 1906

(= Rh. lunulatus Neumann, 1907).

Cette espèce semble peu fréquente, et sa biologie est mal connue pour cette raison. $\mathrm{D}^{\prime}$ une façon générale, elle se trouve presque toujours mêlée en petite quantité à $R h$. simus, dont elle doit avoir les carastéristiques biologiques.

En fait c'est en raison de la faible importance des prélèvements de $R h$. simus que $R h$. tricuspis n'est que rarement rencontré. Au Dahomey, où on récolte $R h$. simus en grande quantité dans la plupart des cas, nous avons pu retrouver à maintes reprises $R h$. tricuspis, espèce qui finalement est présente en de nombreux points de ce territoire.

HOTES

Grands mammifères domestiques et sauvages. DISTRIBUTION

Toute l'Afrique au sud du Sahara.

DISTRIBUTION DE Rhipicephalus tricuspis EN AFRIQUE OCCIDENTALE

Sénégal :

Sangalcam (Rufisque); Niokolo-Koba : guib, Sédhiou (Morel, 1956).
Guinée Portugaise :

Fulacunda : buffle, Bissau : porc (Tendeiro, 1951).

Guinée Française :

Kamayenné (MHNP : Conakry).

Sierra Leone :

Yeria (Zumpt, 1943).

Haute Volta :

Samandéni ; Tiéra (SGHMP : Bobo).

Togo :

Bismarckburg : mouton (Dönitz, 1910 : Rh. glyphis) ; Kpandu (Zumpt, 1943).

\section{Dahomey :}

Cotonou : porc ; Pobé : chien (IPP : Porto Novo); ferme Okpara ; Yérémarou (Parakou) ; Djougou ; Kouandé ; Tanguiéta ; Koni : bovin, mouton (Nikki); Gogonou : mouton (Kandi); Séfon : bubale; Toussou (Banikoara) ; Liboussou (Segbana).

\section{Gold Coast-Ghana :}

Stewart (1935) : localité non précisée.

Nigéria :

Bauchi, Bakanké, Bakeng, Jakiri, Daudawa, Kafinsoli, Mai-Idontoro, Bukwium, Gwarnyo, Wurno, Ovo (Unsworth, 1952).

\section{Oubangui Chari :}

Fort Sibut : porc, chien (IPP) ; Besson (Baboua).

\section{0) Rhipicephalus ziemanni Neumann, 1904}

$C_{c}$ rhipicéphale est une tique de région sahélienne, surtout de forêt. Il vit principalement sur les herbivores sauvages, plus rarement sur les carnivores. On le rencontre parfois sur les animaux domestiques.

- Son aire de répartition se limite à la régicn occidentale d'Afrique (A.O.F., A.E.F., Congo Belge et territoires englobés).

DISTRIBUTION DE Rhipicephalus ziemanni EN AFRIQUE OCCIDENTALE

Sénégal :

Bignona : guib (Villiers, 1955).

Libéria :

Miamu : singe (Bequaert, 1930). 


\section{Sierra Leone :}

Konkoba : chèure ( $\mathrm{CNm})$.

\section{Guinée Française :}

Kongodou, Yrandou (Guéckédou); Didita, Kokota (Nzékoré).

\section{Gold Coast-Ghana :}

Obuasi (Neumann, in tab.).

\section{Côte d'lvoire :}

Yapo : céphalophe d̀ dos noir, antilope royale (Agboville : Villiers, 1955) ; Yapo : potamochère ; Nzida : céphalophe noir (Grand Lahou); Divo; Toumodi : ourébie (IPP); Adiopodoumé : guib ; Koléahinou : céphalophe à dos noir (Soubré); Tai : panthère.

\section{Dahomey :}

Cotonou : porc; Hountagbé (Athiémé).

Nigéria :

Benin, Effon, Ekiti (Unsworth, 1952) ; Mamfé : oanthère, daman (Hoogstraal, 1954).

\section{ARGASIDAE}

270) Argas persicus (Oken, 1818).

\section{BIOLOGIE}

Comme chez tous les Argasidae le nombre des repas des stades n'est pas fixe comme chez les lxodidae, et toujours supérieur à trois.

Les larves seules se gorgent lentement. Ce sont elles qu' on trouve fixées sous les ailes des poulets. Elles y demeurent en moyenne une semaine. Les autres stades se gorgent plus rapidement (1/4 d'heure à 2 heures) selon la températurc et l'état de l'hôte. Les repas ont lieu la nuit, car le photophobie des Argas est très marquée. Le jour ils se réfugient dans les crevasses des murs des poulaillers, derrière les planches, sous les mangeoires etc... La survie est très longue dans les poulaillers désaffectés. Nous en avons conservé de Korhogo en tube de septembre 1955 à novembre 1957 (où ils furent mis à piquer). Il y a deux ou trois stades nymphaux.

\section{HYGROPHILIE}

D'après Bodenheimer (1934), l'optimum des conditions vitales est de $80 \%$ d'humidité relative à $20^{\circ} \mathrm{C}$. La zone où ils se trouvent en A.O.F. ne répond pas à ces données, car la sécheresse y règne une grande partie de l'année, et la température y est la plupart du temps supérieure à $20^{\circ}$. En fait n'ayant pas de phase libre dans la nature, l'argas est toujours protégé du soleil. La proximité des animaux y entretient une certaine humidité, ainsi que l'évaporation de la litière souillée d'excréments. Il semblerait que les argas se raréfient en région guinéenne, sensibles à une trop forte humidité. Ici interviennent les coutumes des éleveurs. Au Sahel, en région soudanienne, les paysans construisent des abris en banco, en hois ou en chaume. Dans le sud (au Dahomey notamment) les poules s'abritent n'importe où, changent de place chaque nuit, dorment sous les hangars, dans des granges : on ne leur construit pas d'abris. En région guinéenne, chez les éleveurs qui ont des poulaillers, des argas sont apparus, d'après les renseignements recueillis. Mais comme il ne s'agissait peut-être pas d'Argus persicus il n'en a pas été tenu compte dans ce rapport.

\section{DISTRIBUTION}

L'argas des poulets est répandu dans le monde entier, à la suite de son hôte. Il ne semble pas que ce soit l'importation de volailles par des européens qui ait amené l'argas en A.O.F. On en trouve dans les villages très reculés. L'arrivée de la tique doit être contemporaine de celle du poulet dans ces régions (par l'est en provenânce d'Asie).

\section{HOTES}

Toutes les volailles de basse-cour peuvent être piquées. Les mammifères domestiques sont parasités plus rarement, et l'homme exceptionnellement (au contraire de ce qui se passe avec $A$. reflexus en Europe).

Les renseignements concernant les oiseaux sauvages sont peu nombreux et d'interprétation difficile : peut-être s'agit-il d'espèces différentes de $A$. persicus dans beaucoup de cas.

Distribution d'Argas persicus en afRIQUe occiDENTALE

$$
\text { Côte d'Ivoire : }
$$

Tiassalé (IPP) ; Korhogo.

$$
\text { Dahomey : }
$$

Kandi.

$$
\text { Haute Volta : }
$$

Gaoua ; Bobo-Dioulasso ; Karankasso (SGHMP': Bobo); Ouagadougou : (Bouet, 1909).

\section{Sénégal :}

Dakar ; Louga ; Saint-Louis (Brumpt, 1909) $(\mathrm{CNm})$; Podor, Thiès, Kaolack, Tambakounda (Rapport Annuel du Service de l'Elevage du Sénégal, 1955). 


\section{Mauritanie :}

Rosso; Boghé ; Boutilimit.

\section{Soudan Français :}

Bamako ct Ścgou : poule, pintade, pigcon, dindon (Rousselot, 1953) ; Nioro ; Djenné (IPP); Gogoro (Douentza) ; Cniminiama ; Tombouctou (IPP) ; Kabara (MHNP).

\section{Niger :}

Dakoro ; Zinder ; Agadès ; In Call (Agadès) ; Maradi : pie piac-piac (l).

\section{Guinée Portugaise :}

Pessuba (Bissau : Tendeiro, 1951, 1952).

\section{Gold Coast-Ghana :}

Tamale (Fulton, 1929) ; sans localité précise (Stewart, 1933, 1934).

\section{Nigéria :}

Mettam (1943 : fide Hoogstraal, 1956).

$$
\text { Tchad: }
$$

El Hamis (MHNP).

\section{Sahara :}

In Salah, Idélès, In Eker (Foley, 1929, 1930) ; Adrar, Timimoun (Foley et Meslin, 1924).

$$
\text { Lybie (Fezzan) : }
$$

Edri (Garibaldi, 1935).

270) Argas reflexus (Fabricius, 1794) (ou espèce voisine).

L'argas du pigeon est rare en Afrique au sud du Sahara. Les quelques récoltes en A.O.F. ne permettent pas d'en esquisser une répartition. Dans le cas des argas de Bessassi, il n'y avait pas de pigeons à proximité (sinon des Columbiformes sauvages); il n'y en avait jamais eu, au dire des villageois (Bessassi est en dehors des grandes voies de passage au Dahomey). Les conditions climatiques de la région soudanienne ne correspondent pas du tout à celles que trouve $A$. reflexus en Europe. Il doit s'agir, en Afrique tout au moins, d'une forme à biologie très nettement adaptée, si ce n'est pas une espèce voisine. La morphologie de nos spécimens est identique à celle de l'A. reflexus de Heisch (1954) du Kénya.

Les références pour l'A.O.F. sont les suivantes: Bamako, Ségou (Rousselot, 1951, 1953) (s'agit-il du véritable reflexus ou de notre espèce?) ; Gaoua : poulail'er (Haute Volta); Bessassi ; poulailler en banco (Nikki, Dahomey).
28\%) Ornithodoros foleyi (Parrot, 1928.)

Décrit du Hoggar (Garet cl Djenoun), s'attaquant a l'homme, au dromadaire (Parrot, 1928; Foley, 1929), au zébu et à la chèvre (Foley, 1929, 1930), il existe peut-être dans l'Air ou l'Adrar des Ifoghas. Un exemplaire a été récolté dans l'Erg Iguidi (Ouahila : IPP). A Chadamès (Lybie), il est dit se gorger sur bovins et gazelles (Tonelli-Rondelli, 1930: O. franchinii). Garibaldi (1935) cite l'espèce de Brak et Edri (Fezzan).

$30^{\circ}$ ) Ornithodoros savignyi (Audouin, 1827).

\section{BIOLOGIE}

La larve éclose mue en nymphe sans prendre de repas, reste immobile. Il y a au minimum quatre stades nymphaux, à repas rapides d'une demi-heure environ. Les adultes prennent plusieurs repas et les femelles pondent à plusieurs reprises. Nous avons observé par $31^{\circ} \mathrm{C}$ des femelles qui se gorgeaient complètement en 20 minutes.

\section{HYGROPHILIE}

$O$. savignyi est une tique des régions subdćsertiques du continent africain, Proche Orient, Indes. En A.O.F. on ne le trouverait pas sensiblement au sud de l'isohyète de $500 \mathrm{~mm}$. Il doit être présent sur tout le pourtour du Sahara. Nymphes et adultes demeurent dans le sable.

HOTES

Ce sont les grands mammifères, principalement le dromadaire, qui semble en avoir été l'agent de dissémination. Les troupeaux de zébus sont attaqués près des gites de nuit, des mares, des puits; les ornithodores sortent alors du sable en grand nombre, piquent rapidement et se laissent tomber. Kassoum Kone (1949) a signalé sur le bétail de Nguigmi des accidents graves, dus probablement à la toxicité de la salive de l'ornithodore. Depuis cette date le même observateur nous a signalé in litt. les mêmes accidents à Gouré.

DistRibition de Ornithodoros savignyi EN AFRIQUE OCCIDENTALE

\section{Mauritanie :}

Bou Tombouskit (Boghé).

\section{Soudan Français :}

Azaouad (région au nord de Tombouctou : Brumpt, 1936): Tin Temarin, Agueni (CBpt); Andéramboukane. 


\section{Niger :}

Nguigmi (Kassoum Koné, 1949 ; Ruusselot, 1951, 1953); Agadès (MHNP); Marandet, Tourouf (Agadès) ; Gouré ; Korgom (Tessaoua); Dakoro ; Laham (Tahoua); Tigueséfen.

\section{Nigéria :}

"Shores of Lake Tchad "(Alcock, 1915 in R.A.E.).

\section{Tchad :}

Bassin sud du Lac Tchad (MHNP $=$ Neumann, 1901 : O. savignyi caecus). Massakori (IPP); Moussoro (IPP) ; Salal (IPP) ; Ouadaī (région d'Abécher : MHNP); Biltine (ColasBelcour et Jacquemin, 1953).

\section{Lybie (Fezzan) :}

Edri(Tonelli-Rondelli, 1932) ; Tmessa, Sciaua, Borg Mzezzem, Uenzerich (Tonelli-Rondelli, 1935) ; Brak (Garibaldi, 1935).

\section{RÉPARTITION DES TIQUES EN A.O.F. EN FONCTION DES ZONES CLIMATIQUES}

La répartition d'un parasite en général n'est pas conditionnée uniquement par la présence de son hôte. Il faut en effet tenir compte, dans le cycle total du parasite, des phases libres dans la nature. Selon leur importance, leur durée, la nécessité d'un hôte intermédiaire ou de conditions microclimatiques définies, seront limitées les chances de maintien de cette espèce.

Les tiques sont des parasites temporaires, la durée, pour la plupart, du repas de sang. Les phases critiques se passent sur le sol, c'est-àdire la ponte, l'éclosion, les mues, moments où l'organisme se trouve d'une extrême sensibilité à l'égard des agents extérieurs. Ce sont ces différences de sensibilité chez les diverses espèces de tiques qui conditionnent en fait leur répartition géographique.

Cette influence est particulièrement nette chez les tiques qui ne sont pas lièes à un seul hôte bien défini. Cette multiplicité ne met que mieux en évidence la dépendance relative par rapport à la qualité des hôtes, ce qui sous-entendl'influence prépondérante des conditions climatiques.

Les tiques du bétail ne sont pas spécifiques. Elles ont des affinités vis-à-vis d'un très grand nombre d'animaux sauvages et s'adaptent rapidement à un animal domestique introduit dans un pays au point de devenir plus abondantes sur cet hôte que sur les hôtes sauvages primitifs.
Dans la défiri.ion des zor ej climétiques ir.tervienr.ent en plus des hauteurs de pluizs annuelles, le nombre de jours de pluies, le nombre de mois secs consécutifs, l'aspect de la végétation, la nature du terrain, les grandes collections d'eau (lacs, marécages, rivières), le régime des vents, l'altitude, etc... En tant qu'expression d'un facteur simple, ce sont les isohyètes qui ont le plus d'utilité pratique en A.O.F. pour juger de la répartition probable ou confirmée des tiques.

En Afrique occidentale, les hauteurs moyennes des pluies sétagent entre $100 \mathrm{~mm}$ et plus de $4.000 \mathrm{~mm}$ au cours de l'année. Une ligne critique importante pour la répartition des tiques est constituée à lisohyète des $500 \mathrm{~mm}$ annuels. Au nord de cet isohyète de $500 \mathrm{~mm}$, les espèccs sont peu nombreuses, mais représentées par de très nombreux individus, adaptés au milieu spécial qu'est le Sahel. Numériquement, c'cst au niveau de cet isohyète 500 que les infestations sont les plus importantes. En allant vers le sud, les espècès se font plus variécs, mais les infestations sont moins massives. Déjà en région soudanienne sud, les infestations sont numériquement peu importantes.

Prenons l'exemple des Hyalomma dont il y a cinq espèces en A.O.F. On ne les trouve couramment qu'en région sahélienne, mais là leur développement numérique y est considérable à un point tel que pour le sujet qui nous occupe, cette zone pourrait être dénommée zone des Hyalomma. A l'intérieur du genre ces espèces manifestent une hygrophilie plus ou moins prononcée, et l'aire d'extension de chacune peut être définie à l'aide de deux courbes isohyètes. Ainsi, $H$. dromedarii est compris entre les courbes de 100 à $500 \mathrm{~mm}, H$. impeltatum entre 250 et $1.000 \mathrm{~mm}, H$. rufipes entre 250 et $1.250 \mathrm{~mm}$, $H$. impressum entre 500 et $1.250 \mathrm{~mm}, H$. truncatum entre 500 et $1.500 \mathrm{~mm}$ (le plus hygrophile : quelques exemplaires sont retrouvés jusquà $3.000 \mathrm{~mm}$ ). Toutes les espèces se fixent à l'état adulte sur les ongulés domestiques et sauvages.

De même Rhipicephalus evertsi (du bétail) et $R h$. cuspidatus (de l'oryctérope) ne se retrouvent qu'en zone sahélienne et soudanienne nord (entre 500 et $1.000 \mathrm{~mm}$ annuels). Ornithodoros savignyi vit dans le sable de toute la région sahélienne sur la bordure du Sahara (entre les courbes de 100 à $400 \mathrm{~mm}$ ).

Par contre d'autres espèces sont localisées en région forestière ou guinéenne : Haemaphysalis parmata, Rhipicephalus ziemanni (des ongulés sauvages) en région forestière ou préforestière : 
Haemaphysalis acidulifer (des ongulés sauvages, parfois sur le bétail), Boophilus annulatus (du bétail) en région guinéenne et soudanienne sud.

Certaines tiques, comme Amblyomma variegatum et Boophilus decoloratus, ne semblent pas limitées par les conditions climatiques audelà du minimum de $400-500 \mathrm{~mm}$ de pluies annuelles. On les retrouve en effet de ces isohyètes jusqu'en région forestière. Elles se fixent sur de nombreux hôtes. Ce sont en fait les tiques les plus abondantes en A.O.F., sauf en région sahélienne, sur le bétail. Il leur faut donc une notable plasticité biologique pour s'accomoder de conditions aussi différentes que le sud du Sahel ou la forêt, et c'est certainement dans ces faibles exigences qualitatives de base qu'est la raison de leur prolifération. Ceci ne veut pas signifier cependant que si on trouve ces deux espèces au Sahel et en forêt, des souches prélevées en forêt se développeront sous le climat du Sahel et vice versa. Il semble plutôt qu'il puisse y avoir une différenciation de telles souches du point de vue biologique, sans qu' il y ait de répercussions appréciables sur la morphologie. La
Ainsi, Rh. simus se sépare en deux sous-espèces : la forme typique, rencontrée entre les courbes isohyètes de 500 à $1.250 \mathrm{~mm}$ de pluies annuelles, et la forme senegalensis, présente à partir des $1.250 \mathrm{~mm}$ jusqu'en forêt (beaucoup plus abondante que la première d'ailleurs). Rhipicephalus sanguineus se présente sous la forme typique dans le Sahel et la zone soudanicnne nord (jusqu'à $1.000 \mathrm{~mm}$ annuels) et sous une forme très ponctuée de moindre taille, à partir de cette limitc jusqu'cn forêt. C'cst du moins l'interprétation personnelle des résultats des prospections en A.O.F. Il ne semble pas que les auteurs aient jusqu à présent souligné ce fait, quoique les différences morphologiques de ces variétés aient été établies. Une différence morphologique, même minime, en corrélation avec des différences plus ou moins profondes dans la biologie d'une souche (ici par rapport à la pluvionétrie) autorise à maintenir la distinction de telles sous-espèces.

En résumé, les diverses zones climatiques isolent en A.O.F. des genres (Hyalomma, Ornithodoros), des espèces ( $R h$. evertsi, $R h$. cus-

\begin{tabular}{|c|c|c|c|c|}
\hline & $\begin{array}{c}\begin{array}{c}\text { Zone } \\
\text { sahélienne }\end{array} \\
250-500 \mathrm{~mm}\end{array}$ & $\begin{array}{c}\text { Zone } \\
\text { soudanienne } \\
\text { nord } \\
500-1000 \mathrm{~mm}\end{array}$ & $\begin{array}{c}\text { Zone } \\
\text { soudanienne } \\
\text { sud } \\
1000-1250 \mathrm{~mm}\end{array}$ & $\begin{array}{c}\text { Zone des } \\
\text { savanes } \\
\text { guinéennes } \\
1250 \text { mm-forêt }\end{array}$ \\
\hline 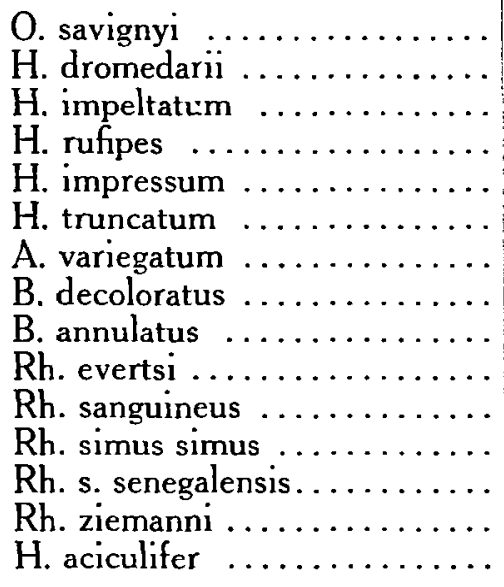 & $\begin{array}{c}++ \\
++ \\
++1 \\
++ \\
+\end{array}$ & $\begin{array}{c}+ \\
++ \\
+ \\
+++ \\
++ \\
++ \\
+ \\
++ \\
++ \\
+\end{array}$ & $\begin{array}{c}+ \\
+\dot{+} \\
+\dot{+} \\
+++ \\
+++ \\
++ \\
++ \\
++ \\
++\end{array}$ & $\begin{array}{l}\frac{1}{1}++ \\
\frac{1}{1}++ \\
\frac{1}{1}+ \\
\frac{1}{1}++ \\
\frac{1}{1}+\frac{1}{1}\end{array}$ \\
\hline
\end{tabular}

mise en évidence de telles souches serait donc nu travail à envisager.

Des faits de la sorte sont d'autant plus probables que c'est sûrement la signification profonde des différences morphologiques qui accompagnent des particularités biologiques chez des Rhipicephalus qui ont une large répartition. pidatıs, Rh. ziemanni, $H$. aciculifer, $H$. parmata, $B$. annulatus, etc...), des sous-espèces ( $R h$. simus simus et simus senegalensis, $R h$. sanguineus sanguineus et sanguineus punctatissimus) et probablement des souches (Amblyomma variegatum et Boophilus decoloratus).

$\mathrm{Si}$ ces tiques multispécifiques peuvent par 
définition s'attaquer aux hôtes les plus variés, il convient de noter que les adultes se gorgent plutôt sur les ongulés, carnivores, etc, tandis que les stades immatures se fixent plutôt sur les oiseaux et les petits mammifères (rongeurs, insectivores, etc...) sauf bien entendu les Boophilus qui effectuent tous leurs stades sur un même hôte (ongulé).

Si on veut établir une liste des tiques dominantes sur le bétail dans chacune des zones climatiques, par ordre de fréquence, nous avons le tableau ci-avant (les croix indiquent l'abondance relative).

(Laboratoire Central de l'Elevage, Dakar, Directeur : P.Mornet).

\section{IV. - BIBLIOGRAPHIE}

Nous citons seulement les références concernant la répartition géographique dans la région qui nous occupe. Pour une bibliographie plus complète se reporter à Hoogstraal. (1956).

Alcock (A.). - (1915) Report of the Entomologist to the London School of Tropical Medicine, 1914, R.A.E., (B), 3 (9), 129.

ANDRÉ (M.) et LAMY (E.). - Les acariens parasites des mollusques (notes complémentaires). J. Conchyliol., 1931, 75, 322.

Augiéras (M.), Draper (W.-E.), Gierzynsky (E.), Besnard (V.) et Monod (Th.). - D'Algérie au Sénégal: Mission Augiéras-Draper, 1927. 1928. Soc. Edit. géogr. mar. col., Paris, 1931, $295 \mathrm{p}$. in $4^{\circ}$. (Atlas de 9 cartes, ibidem, 1930).

Bacelar (A.). - Notas acerca dos Aracnideos do Ultramar Portuguès. Coloq. Jta Invest. colon., Lisboa, 1950, 17, 1-24.

BEAL (W.-P.-B.). - (1920) Report on Live stock Industries of the Northern Territories, GoldCoast, 97 p. R.A.E. (B), 1921, 9 (6), 100-1 ; Trop. Vet. Bull., 1921, 9 (1), 36-50.

Bequafrt (J._C.). - Medical and Economic Entomology. The African Republic of Liberia and the Belgian Congo. Contr. Dep. Trop. Med. Harvard Inst. Trop. Biol. Med., 1930, 2 (5), 797-1001.

Blanc (G.), Goiran (E.) et Baltazard (M.). Observation de deux cas de fièvre boutonneuse à Conakry (Guinée Française) et découverte chez les rhipicéphales de la région du chalcidien Hunterellus hookeri. Arch. Inst. Pasteur Maroc, 1937, 1 (4), 667-76.
Blanc (G.), Goiran (E.) et Baltazard (M.). Idem. Bull. Soc. Path. exot., 1938, 31 (1), 23-8.

Bodenheimer (F.-S.). - Ecological Studies on Some Ticks. Parasit., 1934, 26 (4), 489-95.

Boukt (G.). - Spirillose des poules au Soudan français. Bull. Soc. Path. exot., 1909, 2 (6), 288-91.

Brumpt (E.). - Sur une nouvelle spirochétose des poules du Sénégal produite par Spiro. cheta neveuxi n. sp. Bull. Soc. Path exot., 1909, 2 (6), 285-8.

Brumpt (E.). - Distribution géographique et rôle en pathologie humaine de l'O rnithodoros savignyi. Ann. Parasit. hum. comp., 1936, 14 (6), 640-6.

Buck (G.). - Note sur les tiques des animaux domestiques de Madagascar. Commun. Soc. Sci. méd. Madagascar, février 1948, 2 p.

Campana-Rouget (Y.). - (1954) Mue et croissance chez les Ixodoidea. Bull. Mém. Ecole prép. Méd. Pharm. Dakar, 1952-1953, 1, 213-39.

Colas-Belcour (J.). - Contribution à l'étude de Rhipicephalus (Pterygodes) fulvus Neumann et de sa biologie. Arch. Inst. Pasteur Tunis, 1932, 20, (4), 430-3.

Colas-Belcour (J.) et Jaceuemin (P.). - Détexmination des Ixodidæ recueillis par la mission. Institut de Recherches Sahariennes de l'Université d'Alger. Mission scientifique au Tassili des Ajjer (1949). I. Recherches zoologiques et médicales, 1953, p. 3-4 (tiré à part).

Colas-Belcour (J.) et Rageau (J.). - Tiques de Tunisie : Ixodidés. Arch. Inst. Pasteur Maroc, 1951, 4 (4), 360-7.

Conson (J.-F.). - (1916) Entomological and other Specimens Collected in the Northern Territories, chiefly in the Districts of Wa and Lorha. Rep. Accra Lab., 1915, 30-5.

Corson (J.-F.) et Ingram (A.). - (1923) Rep. med. Dep. Gold-Coast, 1922-1923, pp. 28-9, 65-7 (in LeEson, 1953).

Delpy (L.-P.). - Révision par des voies expérimentales du genre Hyalomma Koch, 1844. Ann. Parasit. hum. comp., 1949 a, 24 (1-2), 97-109.

Delpy (L.-P.). - Essai critique de synonymie du genre Hyalomma Koch, 1844 depuis Linné, 1758. Ann. Parasit. hum. comp., 1949 b. $24(5-6), 464-94$.

Delpy (L.-P.) et Gouchey (S.-H.). - Biologie de Hyalomma dromedarii (Koch, 1844). Ann. Parasit. hum. comp., 1937, 15 (6), 487-99. 
Derbal (Z.) et Balis (J.). - Etude comparée de différents traitements contre les tiques des bovins. Bull. Serv. Elev. A.O.F., 1950. 3 (2-4), 7-13.

Dias (J.-A. Travassos Santos). - Sobre duas entidades do género Haemaphysalis C. L. Koch, 1844. Mem. Estud. Mus. Zool. Univ. Coimbra, 1956, 242, 1 -9.

Dönitz (W.). - Die Zecken des Rindes als Krankheitenubertrager. Sitzungsberichte Gesell. Naturf. Freunde Berlin, 1905, 4, 105-34.

Feldman-Muhsam (B.). - Revision of the Genus Hyalomma.. I. Description of Koch's Types. Bull. Res. Counc. Israel, 1954, 4 (2), 150-70.

Fiasson (R.). - Contribution à l'étude des arthropodes vulnérants du Moyen-Congo. Rev. Sci. méd. Afr. franç. libre, 1943, 2 (2), 125-51.

Foley (H.). - Sur divers arthropodes piqueurs observés au cours de la mission du Hoggar. Bull. Soc. Hist. nat. Afr. Nord, 1929, 20 (2), 47-57.

Foley (H.). - Mceurs et Médecine des Touareg de l'Ahaggar. Arch. Inst. Pasteur Algérie, 1930, 8 (2), 167-287. (Chapitre III. Histoire naturelle, 253-67).

Fulton (A.). - (1929) Ann. Rep. Vet. Dept. for the Financial Year 1929-1930. Appendix I. (GoldCoast Colony). Govt. Print. Office, Accra, 38-41.

Gambles (R.-M.). - (1951) Rep. vet. Dept. Nigeria 1950-1951, pp. 39 et 57 (in HoocstraAl, 1956).

Garibaldi (M.). - Distribuzione geographica degli Ixodidi nelle nostre colonie dell'Africa settentrionale. Arch. ital. Sci. med. colon., 1935, 16 (9), 664-71.

Girard (H.) et Rousselot (R.). - La rickettsiose bovine à Rickettsia bovis au Soudan Français. Bull. Soc. Path. exot., 1945, 38 (3-4), 64-77.

Giroud (P.). Colas-Belcour (J.). Pfister (R.) et Morel (P.-C.) - Amblyomma, Hya'omma, Boophilus, Rhipicephalus d'Afrique sont porteurs d'éléments rickettsiens et néorickettsiens et quelquefois des deux types d'agents. Bull. Soc. Path. exot., 1957, 50 (4), 529-32.

Hoogstraal (H.). - Noteworthy African Tick Records in the British Museum (Natural History) Collections. Proc. Ent. Soc. Wash., 1954,56 (6), 273-9.

Hoogstral (H.). - African Ixodoidea (1956), Vol. 1. Ticks of the Sudan. Research Report NM 005 050. 29.07. U.S. Govt. Printing Office. 1956-0-390800, 1101 pp., 372 fig.

JOYEux (Ch.). - Sur quelques arthropodes récoltés en Haute Guinée française. Bull. Soc. Path. exot., 1915, 8 (9), 656-9.
Kassoum Kone.-- Accidents mortels chez les zébus causés par des piqûres d'ornithodores. Bull. Serv. Elcv. Indust. anim. A.O.F., 1949, 2 (1), 25-6.

KratZ (W.). - Die Zeckengattung Hyalomma Koch. Zeitschr. Parasitenk., 1940, 11 (4), 510-62.

Leeson (H.-S.). - The Recorded Distribution of the Tick Rhipicephalus sanguineus (Latreille). Bull. Ent. Res., 1951, 42 (1), 123-4

LeEson (H.-S.). - The Recorded Distribution of Ornithodoros moubata (Murray) (Acarina). Bull. Ent. Res., 1952, 43 (2), 407-11.

Letson (H.-S.). - Some Notes on the Recorded Distribution of Old World Species of Ornithodoros (Acarina). Bull. Ent. Res., 1953, 44 (3), 517-26.

LEESON (H.-S.). - Further Notes on the Geographical Distribution of Old World Species of Ornithodoros (Acarina). Bull. Ent. Res., 1956, 46 (4), 747-8.

Lloverol (H.), Philippe (J.) et Adjovi (P.). Existence de piroplasmoses du porc en Guinée française. Bull. Serv. Zoot. A.O.F., 1942, 5, 206-9.

MACFIE (J.-W.-S.). - Notes on the Insects Collected at Accra during the Year. Rep. Accra Lab., 1915, 76-9.

Mettam (R.-W.-M.). - (1940) Annual Report of the Veterinary Pathologist. Rep. vet. Dept. Nigeria, 1938, 20-31 (in HoogstraAL, 1956).

Mettam (R.-W.-M.). - (1943) Idem., 1941, 7-10. MetTam (R.-W.-M.). - (1947) Idem., 1945, 12-20. MEtTAM (R.-W.-M.). - (1948) Idem., 1947, 15-24. Mettam (R.-W.-M.). - (1950) Idem., 1948, 58-79.

MetTaM (R.-W.-M.). - (1951) Idem., 1949-50, 32-36.

Minning (W.). - Beitrage zur Systematik und Morphologie der Zeckengattung Boophilus Curtice. Zeitschr. Parasitenk., 1934, 7 (1), 1-43.

Mixisg (W.). - (1935) Zur Kenntnis des Genus Boophilus Curtice. I. Zeitschr. Parasitenk., 1935, 7 (6), 719-21.

Mixing (W.). - Zur Kenntnis des Genus Boophilus Curtice. II. Zeitschr. Parasitenk., 1936, 8 (3), 365-70.

Moody (W.-J.). -- Report on the Veterinary Department for the Year 1921. (Government of the Gold-Coast). Govt. Print. Office, 1922, Accra, 1-18.

MOREL (P.-C.) - - Tiques d'animaux sauvages. Mém. IF.A.N., 1956, 48, Le parc national du Niokolo-Koba (Sénégal), (1), 229-32. 
Neumann (L.-G.). - Révision de la famille des Ixodidés. IV e mémoire. Mem. Soc. Zool. France, 1901, 14 (2-3), 249-372.

Neumann (L.-G.). - Notes sur les Ixodidés, VII. Notes Leyden Mus., 1908, 30. (1), 73-91.

Neumann (L.-G.). - Ixodidae. Tierreich, 1911, 26, 1-169, Berlin.

Neumann (L.-G.). - Un nouveau sous-genre et deux nouvelles espèces d'Ixodidés. Bull. Soc. ent. France, 1913, 38 (5), 147.

NutTall (G.-H.-F.). - Acarina (Ixodidæ), in : Parasitologia Mauritanica. Matériaux pour la faune parasitologique en Mauritanie. Bull. com. A.O.F., 1925, 8 (1), 100-1.

NutTal (G.-H.F.-F) et Warburton (C.). - Ixodidae. II. The Genus Ixodes. Cambridge Univ. Press, 1911, 105-348.

NutTal (G.-H.-F.) et Warburton (C.). - Ixodidae. III. The Genus Haemaphysalis. Cambridge Univ. Press, 1915, 349-550.

Parrot (L.). - Un ornithodore nouveau du Sahara algérien, Ornithodoros foleyi n. sp. Bull. Soc. Path. exot., 1928, 21 (7), 520-4.

Raceau (J.). - Ixodidés du Cameroun. Bull. Soc. Path. exot., 1951, 44 (7-8), 441-6.

Ragead (J.). - Note complémentaire sur les Ixodidae du Cameroun. Bull. Soc. Path. exot., 1953, 46 (6), 1090-8.

Robinson (L.-E.). - Ixodidae. IV. The genus Amblyomma. Cambridge Ĺniv. Press, 1926, $1-302$.

Rousselor (R.). - Hyalomma brumpti Delpy, 1946. Description de la larve et de la nymphe. Biologie. Ann. Parasit. hum. comp., 1948, 23 (1-2), 31-4.

Rousselot (R.). - Ixodes de l'Afrique Noire. Bull. Soc. Path. exot., 1951, 44 (5-6), 307-9.

Rousselot (R.). - Notes de parasitologie tropicale. II. Ixodes. Paris, Vigot Editeur, 1953, $1-135$.

Schulze (P.) et Schlottke (E.). - Bestimmung stabellen für das Zeckengenus Hya!omma Koch, s. str. Sitzungsber. Gesell. Naturf. Rostock, 1930, ser. 3, 2, 32-46.

Simpson (J.-J.). - Entomological Research in British West Africa. I. Gambia. Bull. Ent. Res., 1911, 2 (2), 187-239.

Simpson (J.-J.). - Entomological Research in British West Africa. II. Northern Nigeria. Bull. Ent. Res., 1912 a, 2 (4), 301-56.
Simpson (J.-J.). - Entomological Research in British West Africa. III. Southem Nigeria. Bull. Ent. Res., 1912 b, 3 (2), 137-93.

Simpson (J.-J.). - Entomological Research in British West Africa. IV. Sierra Leone. Bull. Ent. Res., 1913, 4 (3), 151-90.

Simpson (J.-J.). - Entomological Research in British West Africa. V. Gold-Coast. Bull. Ent. Res., 1914, 5 (1), 1-36.

Simpson (J.-J.). - Bionomics of Tsetse and other Parasitological. Notes in the GoldCoast. Bull. Ent. Res., 1918, 8 (3-4), 193-214.

STEWART (J.-L.). - Report on the Department of Animal Health for the Year 1932-1933 (GoldCoast Colony). Govt. Print. Office, Accra, 1933, $11-8$

Stewart (J.-L.). - Report on the Department of Animal Health for the Year 1933-1934 (GoldCoast Colony). Covt. Print. Office, Accra, 1934, $11-9$

Stewart (J.-L.). - Report on the Department of Animal Health for the Year 1934-1935 (Gold-Coast Colony). Govt. Print. Office, Accra, 1935, 12-22

Stewart (J.-L.). - Report on the Department of Animal Health for the Year 1935-1936 (Gold-Coast Colony). Govt Print. Office, Accra, 1936, 9-17.

Stewart (J.-L.). - Report on the Department of Animal Health for the Year 1936-1937 (Gold-Coast Colony). Govt. Print. Office, Accra, 1937, 12-21.

Tendeiro (J.). - Ixodideos da Guiné Portuguesa : Rhipicephalus simus Koch, 1844 e Rhipicephalus sanguineus Latreille, 1806. Bol. cult. Guiné portug., 1946, 1 (3), 397-423.

Tendeiro (J.). - Ixodideos da Guiné Portuguesa : Subfamilia Amblyomminæ NeveuLemaire, 1938. Bol. cult. Guiné fortug, 1947, 2 (7), 617-709.

Tendeiro (J.). - Subsidios para o conhecimento da fauna parasitologica da Guiné. Bol. cult. Guiné porlug., 1948, 3 (11), 639-738.

Tendeiro (J.). - Ixodideos da Guiné Portuguesa : Hyalomma savignyi Gervais, 1844. Bol. cult. Guiné portug., 1949, 4 (14), 319-34.

Tendeiro (J.). - Ixodideos da Guiné Portuguesa : Novos dados acerca da subfamilia Amblyomminæ. Bol. rult. Guiné portug.. 1951 a, 6 (23), 682-90.

Tendeiro (J.). - Ixodideos da Guiné Portugucsa : Nota sobre duas carraças do género Rhipicept:alus. Bol. cult. Griné portug.. 1951 b, 6 (24), 909-28. 
Tendeiro (J.). - Ixodideos da Guiné Portuguesa : Chaves para a identificacâo das carraças guineenses. An. Inst. Med. trop., Lisboa, 1952 a, 9 (1), 195-263.

Tendeiro (J.). - Febre Q. Mem. Cent. Eslud. Guiné portug., 1952 b, 16, 340 pp., Bissau.

TENDEIRo (J.). - Alguns ectoparasitas recolhidos nos Bijagos. Garcia de Orta. Rev. Jia Miss. geogr., Lisboa, 1953, 1 (2), 263-67.

Tendeiro (J.). - Nota sobre um pupipara e um Ixodideo do Cabo verde, Hippobosca maculata Leach, 1817, e Amblyomma variegatum Fabricius, 1794. Rev. Jta Miss. geogr., Lisboa, 1954, 2 (2), 199-203.

Tendeiro (J.). - Sobre alguns Ixodideos dos géneros Hyalomma C.L. Koch, 1844 e Aponomma Neumann, 1899. Bol. cult. Guiné portug., 1955, 10 (39), 319-461.

Theiler (G.). - Ticks in the South African Zoological Survey Collection. IV. Three African Haemaphysalids Parasitic on Domestic Stock. Onderst. J. vet. Sci., 1945, 20 (2), 197-207.

TheILER (G.). - Little Known African Rhipicephalids. Onderst. J. vet. Sci., 1947, 21 (2), 253-300.

TheILER (G.). - Zoological Survey of the Union of South Africa Tick Survey. II. Distribution of Boophilus (Palpoboophilus) decoloratus, the Blue Tick. Onderst. J. vet. Sci., 1949, 22 (2), 255-68.

TheILER (G.). - Zoological Survey of the Union of South Africa Tick Survey. V. Distribution of Rhipicephalus evertsi, the Red Tick. Onderst. J. vet. Sci., 1950, 24 (1-2), 33-6.

Theiler (G.). - Zoological Survey of the Union of South Africa Tick Survey. IX. Distribution of the Three South African Hyalomma or Bontpoots. Onderst. J. vet. Sci., 1956, 27 (2), 239-69.

Theiler (G.) et Robinson (B.-N.). - Zoological Survey of the Union of South Africa Tick Survey. VII. Distribution of Haemaphylasis leachi, the Yellow Dog Tick. Onderst. J. vet. Sci., 1953 a, 26 (1), 83-92.

Theiler (G.) et Robinson (B.-N.). - Ticks in the South African Zoological Survey Collection. VII. Six Lesser Know Rhipicephalids. Onderst. J. vet. Sci., 1953 b, 26 (1), 93-136

Theiler (G.), Robinson (B.-N.), Walker (J.-B.) et WILEY (A.-J.). - Ticks in the South African Zoological Survey Collection. VIII. Two East African Ticks. Onderst. J. vet. Sci., 1956, 27 (1), 83-9.

Tonelli-Rondelli (M.). - Ornithodoros franchinii n. sp., un nuovo argasidae della Tripolitania. Boll. Zool., 1930, 1 (3), 113-5.
Tonelli-Rondelli (M.). - Missione scientifica del Professore E. Zavattari nel Fezzan (1931). Boll. Soc. ent. ital., 1932 a, 64 (6), 106-8.

Tonelli-Rondelli (M.). - Hyalomma nuovi delle colonie italiane. Atti Soc. ital. Sci. nat., 1932 b, 71 (2), 119-25.

Tonelli-Rondelli (M.). - Presenza di Ornithodoros savignyt (Audouin) in Tripolitania. Arch. ital. Sci. med. colon., 1932 c, 13 (2), 117.

Tonelli-Rondelli (M.). - Ixodoidea del Fezzan e della Somalia italiana raccolti dal Prof. E. Zavattari e dal Prof. C. Tedeschi. Atti Soc. ital. Sci. nat., 1935, 74 (3), 239-52.

Unsworth (K.). - The Ixodid Parasites of Cattle in Nigeria, with Particular Reference to the Northern Territories. Ann. Trop. Med. Parasit., 1952, 46 (4), 331-6.

VILLIERS (A.) - Note sur quelques Ixodidae et Gamasidae parasites des vertébrés rencontrés en Afrique occidentale française. Bull. Inst. franç. Afr. noire, 1955, 174 (2), 444-54.

WaLKER (J.). Rhipicephalus pravus Dönitz, 1910. Parasit., 1956, 46 (3-4), 243-60.

Warburton (C.) et NutTall (G.-H.-F.). - On New Species of Ixodidae with Notes on Abnormalities Observed in Ticks. Parasit., 1909, 2 (1-2), 57-76.

Yorke (W.) et Blacklock (B.). - Notes on Certain Animal Parasites on Domestic Stock in Sierra-Leone. Ann. Trop. Med. Parasit., 1915, 9 (3), 413-20.

Ziemann (H.). - Beitrag zur Verbreitung der Blutsaugenden Tiere in West-Africa. Arch. Schiffs. Tropenhyg., 1905, 9 (3), 114-9.

Zumpt (F.). - Vorstudie zu einer Revision der Gattung Rhipicephalus Koch.V.Zur Kenntnis afrikanischer Rhipicephalus Arten. Zeitschr. Parasitenk., 1942 a, 12 (4), 479-500.

ZuMPT (F.). - Vorstudie zu einer Revision der Gattung Rhipicephalus Koch. VI. Rhipicephalus appendiculatus Neum. und verwandte Arten. Zeitschr. Parasitenk., 1942 b, $12(5), 538-51$.

Zumpt (F.). - Vorstudie zu einer Revision der Gattung Rhipicephalus Koch. VII. Rhipice. phalus simus Koch und verwandte Arten. Zeitschr. Parasitenk., 1943 a, 13 (1), 1-24.

Zumpt (F.). - Vorstudie zu einer Revision der Gattung Rhipicephalus Koch. VIII. Rhipice. phalus aurantiacus Neum. und verwandte Arten. Zeitschr. Parasitenk., 1943 b, 13 (1), 102-17.

Zumpt (F.). - Preliminary Study to a Revision of the Genus Rhipicephalus Koch. Doc. Mccambique, 1950, 60, 57-125. 


\section{SUMMARY}

\section{The Ticks in Domestic Animals in French West Africa}

The author presents the results of surveys carried out during the last two years in French West Africa, on the geographical distribution of ticks. He states that this information is not yet complete. In the Order Ixodidae the genera Amblyomma, Boophilus, Haemaphysalis, Hyalomma, Rhipicephalus, Ixodes, and in the Order Argasidae, the genera Argas and Ornithodoros are covered. Wherever possible he gives details on the larval stages, biology, the requirements in humidity, the distribution and hosts.

He has studied the distribution of ticks according to their climatic requirements as he considers that these are different for genera, species, sub-species, and probably even strains.

\section{RESUMEN}

\section{- Las garrapatas de los animales doméstico del Africa Occidental Francesa}

El autor presenta el resultado de los trabajos efectuados durante dos años en A.O.F. con el fin de conocer la repartición geográfica de las diversas especies de garrapatas. Precisa además que éstas informaciones deben ser completadas aún. Cita entre los Ixodidae, los géneros Amblyomma, Boophilus, Haemaphysalis, Hyaloma, Rhipicephalus, Ixodes y entre los Argasidae, los géneros Argas y Ornithodoros. Cada vez que le es posible da precisiones sobre los estados larvarios, la biología, la higrofilia, la distribución y sus húespedes.

Estudia enfin la repartición de las garrapatas en función de las zonas climáticas que regún é lo estima, aislan géneros, especies, sub-especies y probablemente. 